XIV.

\title{
Ein Fall von akuten otitischen Schläfenlappenabszess
}

(induziert durch otitis media suppurativa acuta arteficialis. Einiges zu Statistik der otitisohen Hirnabszesse).

Yon

Dr. Th. Heimann in Warschan.

Willkürlich hervorgerufene Krankheiten des Gehörorgans in Form von Verbrennungen, Verätzungen oder direkter Traumen durch verschiedene seharfe und stumpfe Körper bilden leider bei uns zu Lande unter der militärpflichtigen Jugend ein sehr häufiges Ereignis. Als ich noch im hiesigen Militärhospital die Ohrenabteilung leitete, hatte ich anfangs Gelegenheit, sehr viele solcher Fälle zu beobachten. - Mit der Zeit ist es mir aber gelungen, dieses verbrecherische Handeln aufs Minimum zu beschränken. Wie es scheint, sind aber in den letzteren Jahren, hauptsächlich aber seit dem russisch-japanisehen Kriege, arteficielle Ohrenkrankheiten unter den Militärpflichtigen wieder sehr häufig geworden. Obgleich ich schon mehrere Jahre aus dem Dienst geschieden bin, nötigt mich zu obigem Schluß die wachsende Zahl der arteficiell hervorgerufenen Ohrenkrankheiten, die ich in meiner Privatpraxis zur Behandlung bekomme. Während jeh in den früheren Jahren höchstens zwei bis drei solcher Fälle jährlich beobachtete, behandelte ich im Jahre 1904, und zwar von Oktober bis Ende Dezember 14 Fälle arteficiell entstandener Ohrenkrankheiten, darunter 2 Fälle von unheilbarer Facialisparalyse, 2 Fälle von totaler labyrinthärer Taubheit mit schweren Gleichgewichtsstörungen, die mehrere Monate anhielten und Zerstörung der Paukenböblen, und ein Fall von akuter Nekrose des Warzenfortsatzes mit nachfolgendem Hirnabszeß. Den letzteren übergebe ich der Öffentlichkeit, da es der zweite ähnliche Fall ist, den ich in der mir zugänglichen Literatur fand. Den ersten Fall beschrieb Grubert im Jahre 1891, sein Verlauf war letal; der meinige endigte mit vollständiger Genesung. 
Ende Oktober 1904 besuchte ich den 21 jährigen $A$. A., der seit 6 Wochen an einer rechtsseitigen Otorrhoe litt. Anfangs erklärte mir der Kranke, dal ihm die Ursache des Leidens unlekannt war, später aber gestand er, daß sich das Obrenleiden wach Hereingießen einer ätzenden Flüssigkeit in den äußeren Gebörkanal entwickelte lch fand hinter und über der rechten Ohrmuschel eine taubeneigroße, fiuktuierende Geschwulst, bedeckt mit roter und etwas verdünnter Haut. Die entsprechende Jochbein- und Temporalgegend war ödematoss. Im Trommelfell war ein grober Dofekt vorhanden, die Trommelhöhlenscbleimhaut war rot und verdickt; die Ohreiterung war mäbig. Temperatur $37,0^{\circ}$, Puls 94 in der Minute. Der Kranke klagte über mäBige rechtsseitige Kopfschmerzen. Sein allgemeiner Zustand war befriedigend.

Habitus eines nicht schweren Kranken Zur Operation entschlof sich der Kranke erst den 12. November. Nach Durchtrennen der Weichteile an der üblichen Stelle entleerte sich cin großer subperiostaler Abszeß; im unteren hinteren Teile des Warzenfortsatzes wurde ein nekrotisches Loch konstatiert. Ich entfernte die ganze äulere Wand des Warzenfortsatzes mittels Hammer, Meißel und Knochenzange, alsdann kratzte ich mittels scbarfen Löfels die nekrotischen, mit Eiter und Granulationen gefüllten Warzenzellen bis zum Antrum ans. - Nach Entfernung dieser krankhaften Gewebe kam eine circumskripte Knochenerweichung an der Basis des Warzenfortsatzes zum Vorschein, welche die ganze Dicke des Knochens durchdrang. Diese Stelle wurde auch mit dem scharfen Löffel ausgekratzt, und durch die dadurch entstandene ôfnung zeigte sich die Schädelböble eröffnet Das Loch wurde mit der Knochenzange bis zu $1 \frac{1 / 2}{\mathrm{~cm}}$ im Durchmesser erweitert. Die Dura erwies sich normal. Die Wunde wurde mit Jodoformgaze verbunden. - Der Kranke chloroformierte sich sehr schlecht, bei jeder starken Inspiration entstand bedentende Cyanose, Atemverhaltung und reichliche Schleimabsonderung aus dem Munde. -

Nach der Operation schwand der Kopfschmerz vollständig. Temperatur $37,2^{\circ}$, Pals 78. Subjektiv fühlte sich der Kranke wohl. Nach zweî Tagen fing er an, über mäßigen Kopfschmerz in der Mitte des Scheitels zu klagen. Dabei wurde mäßiges Jieber konstatiert. Temperatur $38,6^{\circ}$, Puls 90 . Es entstand Stuhlyerstopfung, Appetitlosigkeit. Die Zunge war stark belegt. Eine Eisblase auf den Kopf und innerlich Kalomel (0,9) linderten den Kopfschmerz nur für wenige Stunden. Am 16. November steigerte sich der Kopfschmerz bedeutend, der Kranke erbrach zweimal gallige Massen und gleichzeitig wurde bei ihm eine gewisse Benommenheit beobachtet. Fr spricht nichts und antwortet ungern und nur einsilbig suf die an ihn gerichteten Fragen Er ist apathisch, stets somnolent, liegt beständig auf dem Rücken. Bei Veränderung der horizontalen Lage in die sitzende steigert sick die Somnolenz, der Kopfschmerz, und es entsteht dabei recht heftiger Schwindel, so daß der Kranke, nicht gehalten, sofort auf sein Lager zurückfällt. Temperatur 36,20 , Puls 60 .

Am 17. November sind die eben erwähnten Symptome noch deutlicher ausgesprochen. Der Kranke schläft beständig; wird er laut gerufen oder leicht gescbüttelt, öfnet or für einen kurzen Augenblick die Augen, um bald wieder einzuschlafen. Das Bewußtsein ist während der ganzen Zeit vollständig erhalten Totale Appetitlosigkeit und fast unüberwindbare Stublverstopfung. Temperatur $36,6^{\circ}$, Puls 54 .

Am 19. November ist die Somnolenz nicht so stark, als in den früberen Tagen. Im linken Augenhintergrunde wird Hyperamie der Papille, im rechten Stauungspapille mäßigen Grades konstatiert. Die Bewegungen der linken oberen Extremităt sind etwas schwächer, als die der rechten. Temperatur $36,8^{\circ}$, Puls 60 .

Die Wunde hinter dem Obre, die jeden zweiten Tag verbunden wurde, zeigte nichts Abnormes, sie granulierte hübsch; die Ohreiterung war minimal. Der Kranke, der sich bis dahin in einer Privat-Heilanstalt befand, wurde aus materiellen Rücksichter auf eigenen Wunsch in das israelitische Hospital überfürt, um daselbst infolge der sehr deutlichen Symptome eines Hirnabszesses operiert za werden. Die Operation, bei der ich anwesend war, wurde von Dr. Spilrein am 20. Novemuer ausgeführt. Der 
Kranke wurde chloroformiert, das Haar vom ganzen Kopfe wegrasiert und seine Hautfäche desinficiert. Alsdann wurden die reichlichen Granulationen, welche die Knochenwunde ausfüllten, ausgekratzt und die Knochenwunde, die in die Schädelhöhle fübrte, nach oben und hinten erweitert. Die Dura war normal, pnlsierte nicht. Es wurde eine Aspirationsnadel in den Schläfenlappen eingestochen und eine Spritze voll $(10,0)$ dünnen geruchlosen Eiters aspiriert. Bald darauf wurde an dieser Stelle die Dura mit der Hirnsubstanz eingeschnitten und nach Entfernung der Wundränder mittels Kornzange entleerte sicb aus der Tiefe an $30,0 \mathrm{ccm}$ gelbgrünen, geruchlosen, flüssigen Eiters. In die Abszeßhöhle wurde ein Urainrohr aus Glas hineingeschoben und ein steriler Verband angelegt. Bald nach der Operation gab der Puls 82 Schläge in der Minute. Der weitere Krankeitsverlauf war affebril und der Kranke erholte sich sebr rasch. Schon in der dritten Woche nach der Operation konnte er im Zimmer frei herumgeben, und nach vier Wochen wurde er aus dem Hospital entlassen. Die Knochen- und Hautwunde schließen sich nach sechs Wochen; die letzten Verbände werden ihm schon in seiner Wobnung gemacht. Ich bekam den Kranken von neuem zur Beobachtung, als die Wunde hinter dem Ohre vollständig geschlossen war. Die Uhreiterung hielt noch an, aber nach weiteren zwei Wochen sistierte dieselhe beim Gebranche von Aq. oxygenata medicin: 3 proz; der Defekt im Trommelfell hat sich geschlossen und das Gehör ist auf dem rechten Ohre fast zur Norm zurückgekehrt. Bis heute, d. b. Ende Juli, füblt sich der Kranke vollständig wohl, klagt nicht über etwaige Beschwerden, obgleich er sich seit Mitte Januar im Militärdienst befindet, wo er verschiedenen schädlichen Einflüssen, dio mit seinem jetzigen Berufe in unmittelbarem Zusammenbange stehen, ausgesetzt ist.

Wir hatten es also in diesem Falle mit einem akuten rechtsseitigen Schläfenlappenabszeß zu tun, der sich infolge einer akuten arteficiellen Mittelohreiterung, die sich mit akuter Caries des Warzenfortsatzes komplizierte, entwickelte. Als unmittelbare occasionelle Ursache muB wahrscheinlich die Warzenfortsatzoperation angesehen werden, indem bis dahin keine Symptome des Hirnabszesses konstatiert werden konnten. - Der Umstand, daß der Kranke zwei Tage nach der Operation ohne jegliche Ursache zu fiebern und über Kopfschmerz zu klagen anfing, hatte zwar nichts eigentiimliches, das einen Hirnabszeß vermuten ließe, da diese Symptome ebensogut die Anfangsperiode einer allgemeinen Infektion, wie einen frischen Erkrankungsherd im Knoehen bezeichnen konnten; der weitere Verlauf überzeugte aber, daß es die ersten Symptome, eventuell das entzindliche Stadium eines sich entwickelnden Hirnabszesses waren. Die rasch darauf folgende Senkung der Temperatur und Verlangsamung des Pulses und rasches Hervortreten anderer Symptome von erhöhtem intrakraniellem Druck, wie auch, obgleich schwach ausgedrückt, jedoch aber bemerkbare Herdsymptome, boten für die Diagnose keine Schwierigkeiten. - In diesem Falle muh also angenommen werden, daß der Hirnabszel binnen einigen Tagen zur vollen Entwicklung kam.

Schon v. Bergmann macht darauf aufmerksam, daß Ma- 
stoidoperationen gelegentlich einen Hirnabszeb verursachen können. Einen Beweis dafür liefert aueh der unserige Fall. Zufallig hatte ich in derselben Zeit Gelegenheit, noch bei einem anderen Kranken dies zu bestätigen. Fast gleichzeitig operierte ich ein elfmonatliches Kind, bei dem infolge akuter Mittelohreiterung ein Abszeb, durch akute Caries der linken Retroauralgegend hervorgerufen, sich entwickelte. Vor wie nach der Mastoidoperation befand sich das Kind ganz wohl. Einen Tag später erbrach es plötzlich einigemal gallige Massen, es stellte sich zwei Tage anhaltendes, mäßiges Fieber, Obstipation ein, und das Kind wurde von Tag zu Tag somnolenter, wollte nichts zu sich nehmen, das Bewußtsein war aber vollständig erhalten. Nach sechs Tagen zeigten sich anfangs Kontraktionen, später Konvulsionen der rechten oberen Extremität. Der Augenhintergrund erwies sich normal. Zwei Wochen später ging das Kind an Symptomen diffuser Meningitis zugrunde. Obgleich in diesem Falle keine Autopsie gestattet wurde und deshalb nur eine Wabrscheinlichkeitsdiagnose gestellt werden kann, niehtsdestoweniger war der ganze Verlauf für einen Hirnabszeß mit nachfolgender Meningitis diffusa sehr charakteristisch. Auf die Eröffnung des Hirnabszesses wollten die Eltern des Kindes nicht eingeben. Man könnte zwar in diesen beiden Fällen, bauptsächlich aber in dem zweiten, vermuten, daß der $A b s z e ß$ schon vor der Operation vorhanden war, und das Trauma bei der Mastoidoperation nur ein gelegentliches Moment zum Hervortreten seiner Symptome bildete, dagegen sprach aber die Dauer des Ohrenleidens, wie auch der Mangel irgendwelcher Symptome eines Hirnabszesses vor der Operation.

Gelegentlich dieses Falles möchte ich einige Detailfragen aus der Statistik der otitischen Hirnabszesse berühren. Es ist mir nicht darum, neue Gesichtspunkte zu finden, als vielmehr das bekannte Tatsachenmaterial an der Hand einer möglichst großen Zahl von Einzelfällen zu konstatieren. Zwar kann meine Statistik wie überhaupt jede analoge Statistik nur einen relativen Wert besitzen, da sie aber ein verbältnismäßig großes Material in Anspruch nimmt, darf sie vielleicht als näher der Wahrheit entsprechend betrachtet werden, als die Statistiken, die anf kleineren Zablen beruhen.

Aus der mir zugänglichen Literatur der otitisehen Hirnabszesse habe ich 819 Fälle gesammelt. Davon waren mir 645 Fälle in den Originalquellen oder in Referaten zugänglich, und diese sind in Tabelle I ausführlich eitiert. Über die übrigen 174 
Fälle hatte ich nur spärliche Andeutungen oder auch nur bibliographische Notizen zur Verfugung; dieselben bilden Tabelle II. Zur Eruierung der Fragen, die ich zu bertihren gesonnen war, konnten nur die ersten 645 Fälle benutzt werden, das übrige Material nur in sehr beschränkter Weise. Die Literaturquelle ist bei jedem einzelnen Falle angegeben. Wo zwei Quellen erwähnt sind, bezeichnet die erste meistenteils die Originalquelle; die zweite das Referat. - Die Aufsuchung der Originalquellen bot in mehreren. Fällen manche Schwierigkeiten dar, da sie im Referat oder in einer bibliographisehen Notiz irrtïmlich angegeben sind. So z. B. eitiert Körner in seinem Werke: "Die otitischen Erkrankungen des Hirns usw." 3. Anflage 1902, S. 175 den Fall Bakers, dall er nach Hammerseblag eitiert ist und in der M. f. Ohrenh. 1891, Nr. 1 angegeben ist, in Wirklichkeit findet man diesen Fall in der M. f. Ohrenh. 1901, Nr. 1. Den Fall Broca, eitiert, von demselben Autor, babe ich gefunden in den Annales des maladies de l'Oreille 1896 auf S. 424, nicht aber anf S. 409. Okada erwähnt in seiner Arbeit: "Diagnose und Chirurgie des Kleinhirnabszesses" einen Fall von Seblange, der in der Berliner klin. Wochensehrift 1893, S. 677 beschrieben sein soll, aber leider ist ein solcher in dieser Zeitschrift für das ganze Jahr 1893 nicht zu finden. Ioh beschränke mich auf diese zwei Beispiele.

Zum Gegenstand meiner Arbeit habe ich folgende Detailfragen gewählt:

1. Verhältnis der otitischen Groß- zu den Kleinhirnabszessen, wie auch das gegenseitige Verhältnis der Abszesse in den verschiedenen Abschnitten des Großhirns.

2. Verbältnis der otitischen Groß- und Kleinbirnabszesse in verschiedenem Lebensalter.

3. Verhältnis der otitischen Groß- und Kleinhirnabszesse bei verschiedenem Geschlecht.

4. Auf weleher Seite des Grob- und Kleinhirns werden otitische Abszesse ofters angetroffen.

5. Simplicität und Multiplicität der otitischen Hirnabszesse.

6. Häufigkeit verschiedener intrakranieller Komplikationen bei otitischen Hirnabszessen.

7. Häufigkeit der nach akuten und chronisehen Ohreiterungen entstandenen otitisehen Hirnabszesse und ihr gegenseitiges Verhältnis.

8. Heilresultate bei otitischen Hirnabszessen im allgemeinen, 
ohne Berïcksichtigung der Operationsmethode. Verhältnis der Heilresultate bei Groß- und Kleinhirnabszessen, die nach akuten and chronischen Ohreiterungen sich entwickelten.

Barr (1) war der erste, der 76 Fälle otitischer Hirnabszesse sammelte. Derselbe fand:

55 mal Schläfenlappenabszesse,

13 = Kleinhirnabszesse,

$4=$ Grob- und Kleinhirnabszesse,

$2=$ in der Brücke,

$2=$ in den Kleinhirnschenkeln.

Nach Barr gehören 77,62 Proz. den Gehirnabszessen an, davon 72,37 Proz. den Schläfenlappenabszessen.

Nach Rob in (2) sind die Gehirnabszesse die hänfigsten, den zweiten Platz nehmen die Kleinhirnabszesse ein; die seltensten sind die in der Brücke. Auf 131 Hirnabszesse otitischen Ursprungs hat $R_{0}$ b in $101=77,09$ Proz. Großhirnabszesse, $28=21,37$ Proz. Kleinhirnabszesse gefunden; in der Brïeke und im vierten Ventrikel je einen.

In einer älteren Arbeit Körners (3) machen die Sehläfen. lappenabszesse nur 37,3 Proz. aller Hirnabszesse aus. Um diesen Widerspruch mit Barrs Statistik zu lösen, sammelte Körner (4) 100 Fälle von otitischen Hirnabszessen und hat alle im Original nachgesehen. 91 daron sind zur Autopsie gelangt, in den übrigen 9 wurde die Diagnose durch die Operation bestätigt. Bei Betrachtung dieser Fälle überzengte sich Körner, daß es 62 Groß hirnabszesse waren, also 62 Proz., 32 Kleinhirnabszesse und 6 mal Groß- und Kleinhirnabszesse. Der Prozentsatz der Großhirnabszesse bei $K$ örner ist also annähernd derselbe wie bei Barr.

Hes sler (5) sammelte 176 Fälle von otitischen Hirnabszessen, darunter waren 106 Großhirnabszesse, 59 Kleinhirnabszesse, 9 Großund Kleinhirnabszesse und 2 in der Brûeke. Das Verhaltnis der Groß- zu den Kleinhirnabszessen ist 60,22:38,53.

Lueien Piqué und Ferrier (6) geben 119 Fälle von otitisehen Hirnabszessen, die sich folgendermaßen verteilten, an:

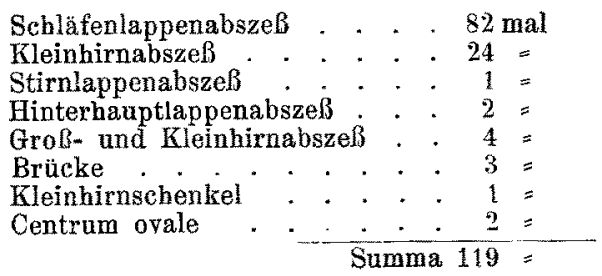


Faßt man in dieser Statistik alle Grob- und Kleinhirnabszesse zusammen, so bekommt man $91=76,47$ Proz. Großhirnabszesse und $25=21,03$ Proz. Kleinhirnabszesse.

J. Robert Weir fand auf 44 Hirnabszesse 24 im Schläfenlappen, 2 im Stirnlappen, 1 im Hinterhauptlappen, 1 in der Bricke und 1 im Vierhügel. Zweimal waren zwei Abszesse vorhanden.

Ein reiches statistisches Material findet man bei Le Fort und Lehmann (7). Diese Autoren sammelten 459 Fälle, darunter waren 327 Großhirnabszesse, 113 Kleinhirnabszesse, 11 Großund Kleinhirnabszesse, 5 in der Brueke, 1 in den Kleinhirnsohenkeln, 1 im vierten Ventrikel. Das Verhältuis der Grob- zu den Kleinhirnabszessen ist $71,24: 24,61$ Proz.

Hier seien noch kleinere Statistiken erwähnt, und zwar:

P it t fand 14 GroB-, 3 Kleinhirnabszesse a. 1 in d. Brücke bei 9000 Antopsien Treitel $=4=3$ Gruber $=39=19$

Meine Statistik, die 645 Fälle enthält, über welche ich genauere Angaben hatte, stellt folgendes Verhältnis dar:

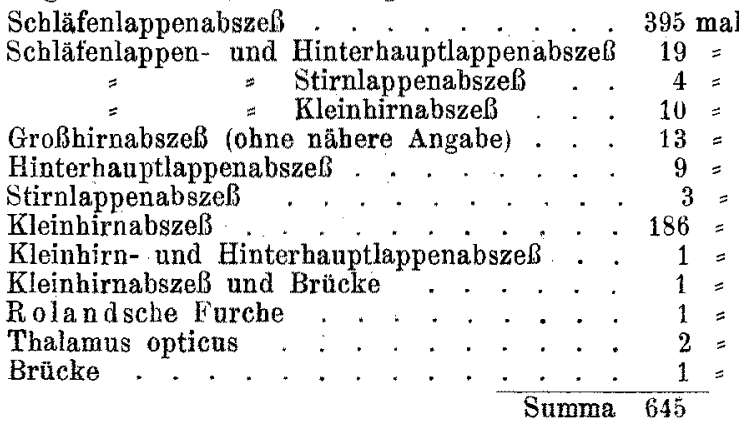

Wir haben also auf 456 Großhirnabszesse 188 Kleinhirnabszesse und einen in der Brücke. Das Verhältnis der ersten zu den zweiten ist $70,68: 29,14$ Proz.

Fassen wir nun die Statistiken versehiedener Autoren zusammen, so ergibt sich folgendes Verhältnis der Grob- zu den Kleinhirnabszessen:

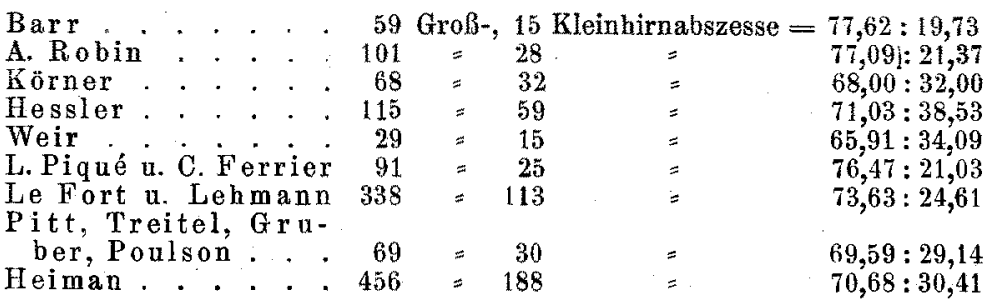


Aus diesen Ziffern kann man mit Sicherheit schließen, daß Grohhirnabszesse durchsehnittlich $2-21 / 2-3$ mal öfter als Kleinhirnabszesse vorkommen.

Aus $\mathrm{Piqu}$ és und meiner Statistik überzengen wir uns außerdem, daß die Stirnlappenabszesse die seltensten unter den Großhirnabszessen sind, und das ohne Unterschied, ob sie vereinzelt oder gleichzeitig mit Sobläfenlappenabszessen zur Entwicklung kommen. Nicht sehr selten ist der Hinterhauptlappenabszeb allein oder gleichzeitig mit Schläfenlappenabszeß kombiniert. Die gleichzeitige Kombination von Groß- und Kleinhirnabszessen erweist sich bei größeren statistischen Zahlen seltener als bei den kleinen Statistiken. Körner fand gleichzeitig Grob-und Kleinhirnabszesse 6 mal auf 100, Hessler 9 auf 176, Le Fort 11 anf 459 und ich 11 auf 645 . Diese Kombination mub also für recht selten betrachtet werden.

Die zweite Tabelle meiner Statistik zeigt auf 174 Fälle 83 Groß- und 91 Kleinhirnabszesse. Die Großhirnabszesse verteilten sich in dieser Gruppe folgendermàßen: Hirnabszeß $38 \mathrm{mal}$; Schläfenlappenabszeb $42 \mathrm{mal}$; Stirn- und SchläfenlappenabszeB $1 \mathrm{mal}$; Hinterhauptlappenabszeß 1 mal und Schläfenlappen- und KleinhirnabszeB 1 mal. Diese Ziffern hätten also im Widerspruch zu den Schlüssen aller anderen Autoren bewiesen, daß3 der Kleinhirnabszeß öfter als der Großhirnabszeß vorkäme. - Dieselben sind aber nicht beweiskräftig, denn sie stellen nur einen Bruchteil der von mir gesammelten Fälle dar. Die allgemeine Ziffer der Groß- and Kleinhirnabszesse meiner Statistik beträgt für die ersten 540, für die zweiten 279 , also fast $2: 1$. Aus demselben Grunde muß die Behauptung Okadas, der sich auf kleine Ziffern von Jansen und Treitel, stützt, daß es zweifelhaft ist, ob Kleinhirnabszesse seltener als Schläfenlappenabszesse vorkommen, für unrichtig betrachtet werden. $0 \mathrm{kada}$ glaubt, daß eine kritiklose Betonung der Tatsache, daB Kleinhirnabszesse viel seltener wären, als Sehläfenlappenabszesse, könnte sogar einmal Grund zur falschen Diagnose geben, indem man Fälle von Kleinhirnabszessen, welche erst später bei der Sektion herausgefunden würden, in Rüeksicht auf jenes Dogma von Wahrscheinlichkeitsgründen zunächst für Schläfenlappenabszesse aussprechen und dann umsonst auf den Schläfenlappen trepanieren könnte. Ioh glaube, daß wo statistische Zahlen von verschiedenen Autoren vorliegen, welche beweisen, daß der Kleinhirnabszeh unbedingt seltener als der Schläfenlappenabszeb vor- 
kommt; da von einer kritiklosen Aeußerung nicht die Rede sein kann.

Das Häufigkeitsverbältnis der Groß- und Kleinhirnabszesse gilt jedoch nur für die Gesamtheit der Fälle. In den versehiedenen Altersklassen ist es nicht gleich. Schon Toy n bee machte die Beobachtung, daß Kleinhirnabszesse bei Erwachsenen relativ häufiger vorkommen, als bei Kindern. Robin bestätigte diese Angabe. Körner fand im ersten Lebens-Dcennium 82 Proz. Grobund 18 Proz. Kleinhirnabszesse; in späteren Decennien 63 Proz. Groß- und 37 Proz. Kleinhirnabszesse. Auch in einer späteren Arbeit bestätigte Körner (8) ein viel selteneres Vorkommen der Kleinhirnabszesse im ersten Lebensdecennium, als bei anderen Autoren, die in ihren Statistiken nur operative Fälle aufgenommen haben, Körner fand nämlich:

Alter Großhirnabszesse Kleinhirnabszesse

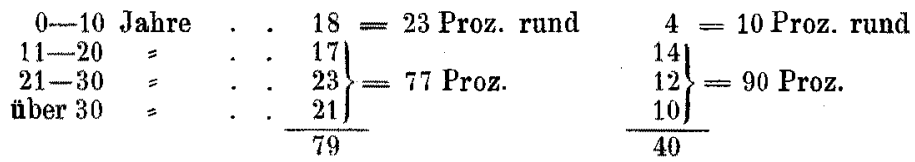

In seiner letzten Arbeit (9) spricht Körner die Meinnng aus, daß dieser Unterschied jetzt nicht mehr so grob sei, wie frühere Statistiken annehmen ließen, wie dies aus dem Vergleich der Altersstatistik von Hammerschlag (10) für den Großhirnabszeß mit der von $0 \mathrm{k}$ a d a für den Kleinhirnabszeß zu ersehen ist:

\begin{tabular}{|c|c|c|c|}
\hline Alter & & $\begin{array}{c}\text { GroBhirnabszeb } \\
\text { (Hammerschlag) }\end{array}$ & $\begin{array}{l}\text { KleinhirnabszeB } \\
\text { (Okada) }\end{array}$ \\
\hline 0 Jahre & & . $27=16$ Proz. rund & $17=11 \mathrm{~F}$ \\
\hline $\begin{array}{l}-20= \\
-30=\end{array}$ & & $\left.\begin{array}{l}47 \\
51\end{array}\right\}=84 \operatorname{Proz}$ & $\left.\begin{array}{l}56 \\
45\end{array}\right\}=89$ Proz \\
\hline 30 & & $44)$ & 28 \\
\hline
\end{tabular}

Nach Röpke (11) waren auf 123 Großhirnabszesse im

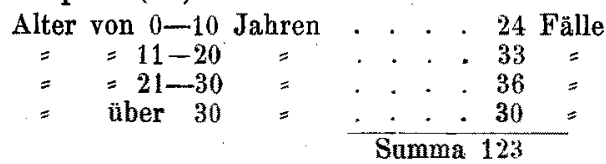

K och (12) fand folgende Zahlen der Kleinhirnabszesse im verschiedenen Alter, und zwar:

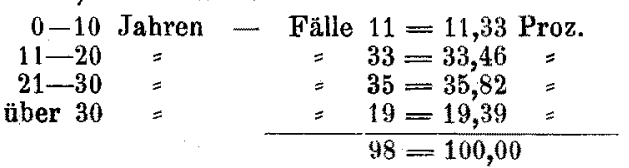

Auf Grund dieser Zusammenstellung spricht sich $\mathrm{Koeh}$ aus, daß die Kleinhirnabszesse im ersten Decennium ziemlich selten 
sind; im zweiten und dritten Decennium am häufigsten und nach dem 30. Lebensjahr am seltensten vorkommen. $0 \mathrm{kada}$ meint, dab man aus diesen Ziffern keinen Scblub ziehen kann, was das numerische Verbältnis zwischen Groß. und Kleinhirnabszessen in den verschiedenen Lebensaltern betrifft, denn die von $\mathrm{Koch}$ gesammelten Fälle von Kleinhirnabszessen entsprechen der Anzahl nach gar nicht den Fällen von Grobhirnabszessen von Körner.

Hessler (13) fand bei der Nachforsehung sämtlicher in der Literatur gefundenen otitischen Hirnábszesse folgende Zahlen:

Alter

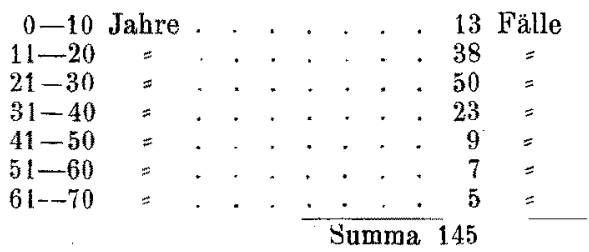

Bei 13 Kindern in dem ersten Decennium waren 8 mal Großund 5 mal Kleinhirnabszesse.

Auf Grund dessen hat er darauf aufmerksam gemacbt, daß die Körnersehe Annahme von der Seltenheit der Kleinhirnabszesse bei Kindern widerlegt ist, wenn man die Gesamtzahlen aller Fälle von otitischen Hirnabszessen und die Differenz zwischen Groß- und Kleinhirnabszeh im kindlichen Alter vergleicht.1)

Um sich zu überzeugen, auf welcher Seite die Wahrheit stehe bei diesen sich widersprechenden Resultaten, hat $\mathrm{Okada}$ die von ihm selbst in der Literatur gesammelten Fälle statistisch zusammengestellt und hat folgendes erhalten:

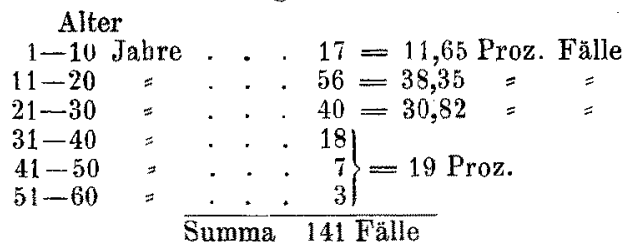

Ok ad a glaubt also nach dieser Tabelle im Rechte zu sein, daß Kleinhirnabszesse wirklich im Alter unter 10 Jahren am seltensten sind, aber diese relative Seltenheit des Kleinhirnabszesses bei kleinen Kindern sei nicht so auffallend und nicht so wichtig, wie Körner behauptet. $0 \mathrm{kada}$ kennt bei

1) Wie ich oben erwähnte, hat Körner in seiner letzten Arbeit diesen Standpunkt aufgegeben. 
Kindern unter 4 Jahren 2 Fälle von Kleinhirnabszeß und von 4 Jahren an 4 Fälle. Nach $0 \mathrm{k}$ a da ist der Prozentsatz der Kleinhirnabszesse bei kleinen Kindern viel größer, als der von Körner, sowie als der Prozentsatz der gesamten Mischformen des otogenen Hirnabszesses, auch ist der Prozentsatz der Grobhirnabszesse bei kleinen Kindern zu groß von Körner angegeben. Er stellt dies Verhältnis in folgender Tafel dar:

$\begin{array}{ccccc}\text { Alter } & \begin{array}{c}\text { Okada } \\ \text { (Kleinhirn- } \\ \text { abszeB) }\end{array} & \begin{array}{c}\text { Körner } \\ \text { (Kleinhirn- } \\ \text { abszeB) }\end{array} & \begin{array}{c}\text { Hessler } \\ \text { (Misch- } \\ \text { formen) }\end{array} & \begin{array}{c}\text { Körner } \\ \text { (Großhirn- } \\ \text { abszeß) }\end{array} \\ 0-10 \text { Jabre } & 11,65 \text { Proz. } & 10 \text { Proz, } & 8,96 \text { Proz. } & 22,78 \text { Proz. } \\ 11-20= & 38,35= & 35= & 26,20= & 21,51= \\ 21-30= & 30,82= & 30= & 34,48= & 29,11= \\ \text { uber } 30= & 19,15= & 25= & 30,34= & 26,58=\end{array}$

Kleinhirnabszesse sind nach $0 \mathrm{kad}$ a ebenso selten bei Kindern wie bei älteren Leuten (über 40), am öftesten sind sie im zweiten und dritten Decennium (69,17 Proz.) oder genauer bis zu Ende des vierten Decenniums. Deshalb stimmt $0 \mathrm{kada}$ mit $\mathrm{K}$ örner nicht überein, daß die Seltenheit der Kleinhirnabszesse für kleine Kinder typisch ist, und deshalb kann dieses Moment diagnostisch nicht verwertet werden.

In meiner Statistik, in der nur bei 535 Fällen das Alter angegeben ist, erhielt ich folgende Ziffern:

$\begin{array}{cccc}\text { Alter } & \text { Großhirnabszesse } & \text { Kleinhirnabszesse } & \text { Summa } \\ 0-5 \text { Jahre } & 14=2,62 \text { Proz. } & 6=1,12 \text { Proz. } & 20=3,74 \text { Proz. } \\ 6-10= & 41=7,66= & 10=1,88= & 51=9,54= \\ 11-15= & 53=9,91= & 29=5,42= & 82=15,33= \\ 16-20= & 55=10,28= & 28=5,23= & 83=15,51= \\ 21-30= & 29=24,11= & 38=71,10 \Rightarrow & 167=31,21= \\ 31-40= & 54=10,09= & 13=2,43= & 67=12,52= \\ 41-50= & 30=5,61= & 8=1,50 \Rightarrow & 38=7,11= \\ 51-60= & 19=3,55= & 3=0,56= & 22=4,11= \\ 61 \text { u. höher } 3=0,36= & 2=0,37= & 5=0,43= \\ & 398=74,39 \text { Proz. } 137=25,61 \text { Proz. } & 535=100 \text { Proz. }\end{array}$

Bei 59 Fällen von Großhirnabszeß und bei 51 von Kleinhirnabszeb war das Alter nicht angegeben. -

Aus den von mir erhaltenen Ziffern ergibt sich: 1. Dalo otitische Hirnabszesse im allgemeinen nach dem 60. Lebensjahre selten vorkommen, wahrscheinlich deshalb, weil dieses Alter durchschnittlich seltener erreicht wird; den zweiten Platz nehmen in dieser Hinsicht die ersten 5 Lebensjahre ein. 2. Die größte Häufigkeit der Groß- und Kleinhirnabszesse kommt auf das dritte Decennium, eventuell auf das zweite und dritte. 3. In dieser Statistik ist die Ziffer der Kleinhirnabszesse bei Kindern vom 6. bis zum 10. Lebensjahre eine bedeutend geringere, als die der Großhirnabszesse in demselben Alter, sie kann aber kaum 
für etwas Eigentümliches für dieses Alter betrachtet werden. Das Verhältnis der Groß- zu den Kleinhirnabszessen im ersten Decennium zu den übrigen Lebensjahren fällt nach meiner Statistik aus:

Großhirnabszesse 10,28 Proz, Kleinhirnabszesse 3 Proz. im ersten Decennium $64,11=22,61$ Proz. in den übrigen Dec. also im ersten Decennium wie auch später annähernd wie $3: 1$. -

Das Verhältnis der otitischen Hirnabszesse bei verschiedenem Gesehlechte ist bei allen Autoren fast das gleiche. - Alle, mit wenigen Ausnahmen, haben sich überzeugt, daß beim männlichen Geschlechte die otitischen Hirnabszesse zweimal so oft vorkommen, als beim weiblichen. Körner fand auf 61 Gehirnabszesse mit Geschlechtsangabe 43 Männer und 18 Weiber, und auf 30 Kleinhirnabszesse 18 Männer und 12 Weiber.

Gruber (J. Pollak) (14) fand reine Gehirnabszesse . $32 \mathrm{mal}$, davon waren 26 Männer 6 Weiber komplicierte $=7$ reine Kleinhirnabszesse $13=$ komplicierte $=$

$6=$

Hessler fand auf 151 Fälle von otitischen Hirnabszessen 93 Männer und 58 Weiber.

Koeh sammelte 102 Fälle von Kleinhirnabszessen, darunter waren 66 Männer und 36 Weiber. Derselbe fand im ersten Decennium 4 Männer und 6 Weiber.

$0 \mathrm{kada}$, der 165 Fälle von Kleinhirnabszessen sammelte, fand 107 Männer und 22 Weiber, in den übrigen Fällen war das Geschlecht nicht angegeben, es war nur "Kind" erwähnt.

Röpke fand in seiner Statistik der Großhirnabszesse 83 Männer und 41 Weiber.

Bei Pitt sind 11 Männer und 7 Weiber und bei Piqué 21 Männer und 1 Weib notiert. -

Meine Statistik stellt folgende Ziffern dar:

Lokalisation des Abszesses

\begin{tabular}{|c|c|c|c|c|}
\hline & & & & \\
\hline Schlâfenlappen : . . . - . & 258 & 58 & 11 & 35 \\
\hline Schläfen- und Finterhauptlappen & 14 & 4 & - & 1 \\
\hline$=\quad=$ Stirnlappen & & & - & \\
\hline Schläfenlappen und Kleinhirn & 1 & 7 & - & 2 \\
\hline Hirnabszel (obne nähere Angabe) & 7 & 3 & - & \\
\hline Hinterhauptlappen . . . . . & 6 & 1. & - & 2 \\
\hline Stirnlappen & 1 & 1 & - & i \\
\hline Kleinhirn & 117 & 33 & 4 & 32 \\
\hline und Hinterhauptlappen & - & - & - & 1 \\
\hline$=$ Bricke. & - & 1 & - & - \\
\hline Brücke . + · . & 1 & - & - & - \\
\hline Rolandsche Furche . . & 1 & - & - & 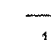 \\
\hline Thalamus opticus & 1 & - & - & 1 \\
\hline
\end{tabular}


Aus diesen Ziffern ist zu ersehen, daß so die Groß- wie die Kleinhirnabszesse bei Männern dreimal so oft, als bei Weibern vorkommen.

Was die Hänfigkeit der otitisehen Hirnabszesse bei versehiedenem Gesehlechte in verschiedenem Alter betrifft, habe ich folgendes Verbältnis gefunden:

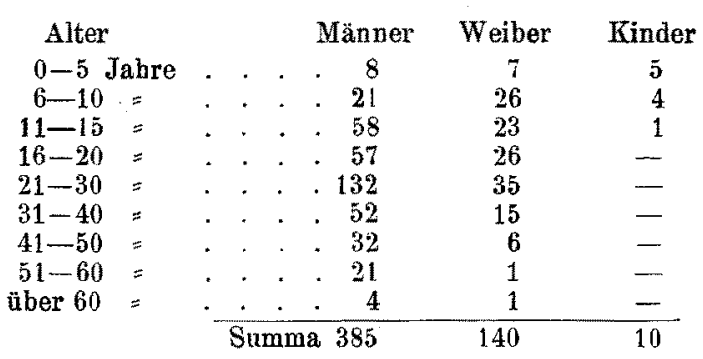

Im ersten Decennium sind also die otitisehen Hirnabszesse etwas öfter beim weiblichen, als beim männlichen Gesehlechte. In einem jeden der ubrigen Decennien ist das Verhältnis umgekehrt. Der gröBte Unterschied fällt in das zweite und dritte Decennium aus. Nach dem vierten Decennium werden die otitisehen Hirnabszesse beim weiblichen Gesebleeht immer seltener.

Wie stellt sieh das Verhältnis der rechtsseitigen zu den linksseitigen otitisehen Hirnabszessen dar?

R. Meyer erwähnt in seiner unter Biermers leeitung verfaßten Dissertation (Zürich 1867), daß von 18 infolge von Felsenbeincaries entstandenen Hirnabszessen $14 \mathrm{mal}$ die rechte und nur 4 mal die linke Hemisphäre befallen war. Dieselbe Erfahrung haben auch Toynbee, Gull und Sutton, Sehwartze (15), H a guen in (16) und Körner (17) gemacht. Letzterer fand auf 151 verwertbare Falle, wo es sich $67 \mathrm{mal}$ um Hirnabszeß $\mathrm{B}$ handelte, unter 31 Fällen von Großhirnabszeß, wo sich die Erkrankung rechts und nach rechtsseitigem Ohrenleiden 18 mal, links und nach linksseitigem Ohrenleiden $12 \mathrm{mal}$, und beiderseits nach einseitigem Obrenleiden 1 mal vorfand. Unter 19 Fällen von Kleinhirnabszessen war derselbe reehts 10 mal, links 8 mal und beiderseits nach beiderseitigem Ohrenleiden $1 \mathrm{mal}$. Die Kombination von Groß- und Kleinhirnabszeß fand sich rechts 2 mal, links und doppelseitig je $1 \mathrm{mal}$. Im ganzen handelte es sich in 39 Fällen um rechtsseitigen, in 25 Fällen um linksseitigen und in 3 Fällen um doppelseitigen Hirnabszeß. Großhirnabszeß war 22 mal rechts und 16 mal links; Kleinhirnabszeb 15 mal reehts und 8 mal links. 
In allen 151 Fällen war die Hirnkomplikation rechts 90 mal, links 51 mal und doppelseitig 4 mal. -

R. Meyer suchte die Ursache des häufigen Vorkommens des Hirnabszesses auf der rechten Seite durch das häufigere Vorkommen der rechtsseitigen Otorrhöen zu erklären. Die Statistik erwies aber, daß dem nicht so ist. Körner erklärte diesen Umstand durch anatomische Verschiedenheiten der beiden Schädelhälften, die das Übergreifen einer im Sebläfenbein verborgenen Erkrankung auf Meningen, Sinus und Hirn rechts leichter zustande kommen lassen, als links. Diese anatomische Verschiedenheit besteht darin, dab auf der rechten Seite die Fossa sigmoidea im Sulcus transversus weiter nach vorn und außen in die Basis der Felsenbeinpyramide und in den Warzenfortsatz hineindringt, als links (77 Proz.). Auf der rechten Seite ist also die Knochenwand, welche ein Empyem, ein Cholesteatom, einen cariösen Herd von den Meningen, dem Sinus transversus und dem Gehirn trennt, in 77 Proz. der Fälle dünner, als links. Und des. halb können auf der rechten Seite krankhafte Prozesse in die Schädelhöhle leichter eindringen, oder durch die Blut- und Lymphbahnen dieselbe eber erreichen, als links. Körner ging noch weiter und sprach die Meinung aus, daß, indem bei Brachycephalen der Sinus transversus auf beiden Seiten der Warzenund Paukenhöhle näher liegt, als bei Doliehocephalen, und daß der Boden der mittleren Schädelgrube bei Brachycephalen tiefer steht als bei Dolichocephalen, deshalb liege bei Brachycephalen die Unterfläche des Schläfenlappens und die Vorderfläche des Kleinhirns mit ihren Meningen und Sinus näher dem Antrum mastoideum und der Paukenhöhle, als bei Dolichocephalen. Auf Grund dieser Tatsachen und statistischen Nacbweise kommt Körner zum Sehluß, daß bei Brachycephalen mit Ohreiterungen letale Folgeerkrankungen leichter zustande kommen, als bei Dolichocephalen. Diesen Standpunkt bemïht sicb Körner in einer späteren Arbeit aufrecht zu halten.

Diese Meinung der oben erwähnten Autoren in Betreff auf den Sitz des Hirnabszesses wird in neuerer Zeit angefochten und durch entsprechende gröBere Zahlen für irrtümlich betrachtet. $\mathrm{R} \not \partial \mathrm{pke}$ hat in seiner Statistik auf 66 rechtsseitige 67 linksseitige Großhirnabszesse gefunden. Er bemüht sich, diesen Umstand dadurch zu erklären, daß seine Operationstabelle nur Grobhirnabszesse umfaßt and die linksseitigen Großhirnabszesse durch das bei ihnen in vielen Fällen rorkommende Herdsymptom der 
Aphasie leichter diagnostiziert und infolge dessen häufiger operiert werden. Denn von 67 linksseitigen Abszessen hatten 52 Fälle Funktionsstörungen infolge Kompression des Gehirns, darunter 38 Fälle, in denen die Kranken Sprachstörungen hatten, während bei den 66 rechtsseitigen Abszessen nur 32 vor der 0 peration Herdsymptome aufwiesen. Von den linksseitigen Abszessen bestand in 12 Fällen als einziges Herdsymptom "Aphasie". Von 135 operierten Grobhirnabszessen sind 84 Fälle $=62,2$ Proz. auf Grund von Herdsymptomen operiert worden. -

Pitt erklärt das Übergewieht der Hirnabszesse auf der rechten Seite als rein zufällig; auberdem findet er in seiner Statistik diesen Unterschied nicht sehr erheblich, denn auf 18 Fälle von Hirnabszeß, wo die Seite angegeben ist, waren 10 rechtsseitig und 8 linksseitig. - Hammersohlag fuhrt 85 rechtsseitige und 96 linksseitige operierte Schläfenlappenabszesse an.

Koch (18) fand, daß der Kleinhirnabszeß keine der beiden Hemisphären in auffallender Weise bevorzugt, indem er unter 103 Kleinbirnabszessen 53 rechts und 48 links sammelte, während in 2 Fällen je ein Abszeh in beiden Hemisphären vorhanden war.

Okada, der 165 Falle von KleinhirnabszeB sammelte, fand 73 reehts, 78 links und 2 Fälle, wo in jeder Hemisphäre ein Abszeb sich befand. Oka da sebließt daraus, dab für den Kleinhirnabszel beider Hemisphären dieselbe Häufigkeit konstatiert ist.

Aber schon Lebert hat die Meinung ausgesprochen, daß die linke Kleinhirnhälfte für Abszesse mehr disponiert ist, als die rechte, indem er unter 12 Fällen 8 auf der linken Seite fand.

Nach Gustav Custers Zusammenstellung sind beide Hirnhälften ungefähr gleich häufig. Auf 31 Großhirnabszesse waren 16 rechts und 15 links; auf 17 Kleinhirnabszesse waren 8 reehts und 9 links.

Körner tritt den Statistiken derer entgegen, die seinen Standpunkt von dem häufigeren Vorkommen der rechtsseitigen Hirnabszesse nicht bestätigen konnten. In dieser Hinsicht drückte er sich folgendermaßen aus: "Was die (von Körner) genannten Autoren übereinstimmend gefunden haben, wird neuerdings auf Grund größerer Zahlen bestritten. Damit ist aber die Frage noch nicht endgültig entschieden, denn die Zahlen der alten Autoren gründen sich ausschlieflich auf Sektionsbefunde, während die neuen Statistiken vorzugsweise, zum Teil aussohließlich, die operierten Fälle heranziehen. Die alten und neuen Zahlen sind also nicht gleichwertig. 
Was zunächst die Schläfenlappenabszesse betrifft, so ist es klar, daß die nach den Sektionsstatistiken der voroperativen Zeit selteneren linksseitigen, infolge der ibnen eigentümlichen erst in der operativen Aera genauer beachteten Sprachstörungen, neuerdings öfter und frühzeitiger erkannt werden, als die häufigeren rechtsseitigen. Sie werden deshalb anch bäufiger mit Erfolg operiert und die so geheilten Fälle werden dann - hier wie überall öfter publiziert, als die Mißerfolge.

Bei den Kleinhirnabszessen, deren Lage in den beiden Hemisphären keine verschiedenen Symptome macht, wird die größere Häufigkeit der rechtsgelegenen allein durch einen anderen Umstand verdeckt, der aber auch die heutige entsprechende Statistik der Schläfenlappenabszesse beeinträchtigt. Wo immer in der Heilkunde ein neuer Satz aufgestellt wird, erhebt sich alsbald eine Reaktion; die nicht zur Regel stimmenden Einzelfälle werden als wertvoll veröffentlicht, die die Regel bestätigenden als selbstrerständlich vernachlässigt. Dies geschieht bald in der ausgesprochenen Absicht, seltenere Vorkommnisse zu registrieren, bald ohne Angabe dieses Grundes, vielleicht auch manchmal halb unbewußt. So häufen sich die Fälle, die dann gesammelt, die Regel umzustoben scheinen.

Den AnstoB zu einer solohen Bewegung in der hier besprochenen Frage gab der von mir geführte Nachweis, daß nicht nur, wie sechs hervorragende Forseher schon vor mir widerspruchslos nachgewiesen hatten, die otitischen Hirnabszesse, sondern auch die Sinusphlebitiden und Meningitiden häufiger dureh eine rechtsseitige Ohreiterung entstehen, als durch eine linksseitige, sowie der erbrachte Nachweis, warum dies geschieht. - So sind wir bereits zu Statistiken gelangt, die mehr linksseitige als rechtsseitige Groß- und Kleinhirnabszesse verzeichnen." -

Nach alledem soheint es Körner richtiger, vorläufig bei der alten Lehre festzuhalten. Er fand auf

106 Großhirnabszesse $59=55,66$ Proz. rechts and $47=44,34$ Proz. links

54 Kleinhirnabszesse $37=68,52=\quad=\quad=17=31,48$

Meine Statistik bat folgende Ziffern ergeben:

Sitz des Abszesses

Schläfenlappen

Schlö... 160

Schlafen- und Hinterhauptappen . 2

Schläfenlappen und Kleinhirn . . 3

Hirnabsze 3 . . . . . . 3

Hinterhauptlappen . . . . . . 6 links

188

8

2

5

4

3
Seite unbekannt

47

2 


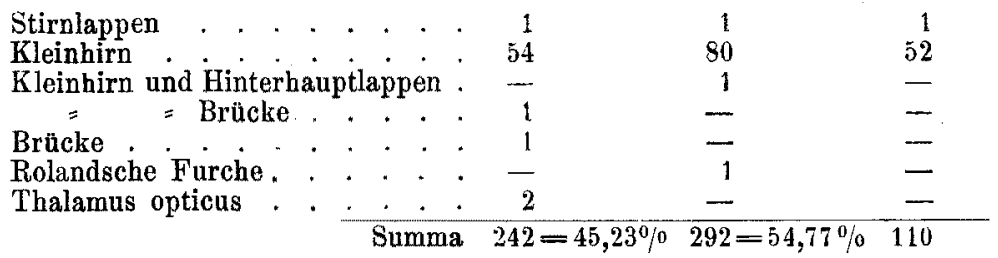

Diese Statistik, die sich auf die größten bisher bekannten Ziffern stützt, zeigt, daß die linke Hemisphäre bei Groß- und Kleinhirnabszessen nicht nur nicht seltener, sondern sogar öfter als die rechte von Hirnabszessen befallen wird. Davon will ich aber nicht den Schluß folgern lassen, daß die rechtsseitigen Hirnabszesse seltener als die linksseitigen vorkommen, und möehte dies nur als reinen Zufall betrachten, ebenso wie es reiner $\mathrm{Zu}$ fall ist, dab in gewissen Statistiken die rechte Seite überwiegt. Nach meiner Ansicht, die durch versehiedene Statistiken ihre Bestätigung findet, hat keine der beiden Grob- wie Kleinhirnhemisphären eine besondere Disposition zu otitisehen Hirnabszessen. - Um mich noch entschiedener über die Richtigkeit eines solchen Standpunktes zu überzeugen, sammelte ich die Fälle von doppelseitiger Otorrhoe, und fand auf 35 Fälle von otitischen Hirnabszessen $18 \mathrm{mal}$ die rechte Seite und $17 \mathrm{mal}$ die linke befallen, also fast das gleiche Verhältnis in beiden Hemisphären. Sollte wirklich die rechte Seite zum Hirnabszeß mehr disponiert sein. als die linke, so würde dieser Umstand bei doppelseitigen Otorrhöen am deutlichsten hervortreten. - Die Ansicht Körners über die Lokalisation des Hirnabszesses, und die von ihm dafür angegebenen anatomischen Bedingungen sind nicht stichhaltig und müssen endgültig aufgegeben werden.

Ioh glaube, daß es den Autoren, die andere Verhältnisse als Körner und die von ihm eitierten Autoren gefunden haben, speciell nicht darum zu thun sein konnte, nur die Ansichtspunkte anderer zu bestreiten, und daß dieselben mit einer idea praeconcepta zur Bearbeitung dieses Gegenstandes nicht schritten. Es ist ja ganz natürlich, wenn ein neuer Satz, eine neue Theorie, eine neue Hypothese in der Heilkunde, wie in allen tbrigen Wissenschaften auftaucht, daß andere Forseher von der Richtigkeit oder Irrtümlichkeit dieser nenen Anschaung sich tuberzengen wollen und müssen; es konnte also mit der Frage der Lokalisation der otitischen Hirnabszesse, ob dieselben rechtsseitig oder linksseitig öfter vorkommen, nicht anders der Fall sein. Daß sechs andere Forscher dieselben Verhältnisse wie Körner fanden, 
kann doch nicht als unfehlbares Dogma, an dem zu rütteln verboten ist. betrachtet werden. Ich habe die feste Überzeugung, daß die Ärzte, welche die Anschauung $K \ddot{\partial}$ rners nicht teilen können, nicht den mindesten Gedanken hatten, nur solche Fälle zu sammeln, die darauf ausgingen, die Körn ersehe Anschaunng zu ersehüttern. Verschiedene gröbere Statistiken, und vorzugsweise die meinige, beweisen, daß die rechte Hemisphäre kein Lieblingsort für otitische Hirnabszesse ist. -

Die Meinung Körners, daß Sektionsstatistiken einen größeren Wert als Operationstatistiken haben, scheint nicht ganz gerechtfertigt zu sein. Es ist unbegreiflich, weshalb ein durch Operation entdeckter Hirnabszeß zur Zusammenstellung einer Statistik minderwertig ist, als die zur Sektion gelangten Fälle. -

Bei statistischen Zusammenstellungen ist die Ziffer entscheidend. Man konstatiert eine gewisse Zahl rechts- und linksseitiger Hirnabszesse, und daraus wird der Schlnß gezogen, welehe Seite öfter befalien wird. Ich habe alle mir zugänglichen Fälle, so die operierten wie die nicht operierten gesammelt, und aus ihrer Zusammenstellung habe ieh erst gewisse Sehliisse gezogen. Daß viele unglückliche Fälle verheimlicht werden, unterliegt keinem Zweifel; das gilt aber ebenso gut für die rechtsseitigen, wie für die linksseitigen Abszesse. Ich möchte mir erlanben, im Gegensatz zu Körner die Vermutung auszusprechen, daß eher linksseitige operierte Fälle, die leichter diagnostizierbar sind, verheimlicht werden, als die rechtsseitigen und die Kleinhirnabszesse.

Die otitischen Hirnabszesse treten in der Mehrzahl der Fälle vereinzelt auf. Körner fand unter 100 Fallen 6 mal je einen Abszeb im Schläfenlappen und im Kleinhirn. - Im Sehläfenlappen allein fand er 5 mal Doppelabszesse auf 62 Fälle, also 8,5 Proz., und Doppelabszesse im Kleinhirn 4 mal auf 32 Fälle, was 12.5 Proz. ausmacht. Nach Körner können auch mehrere Abszesse nebeneinander bestehen und gewöhnlich sind sie miteinander in Verbindung.

Hessler sammelte in seiner Statistik $14 \mathrm{mal} 2$ Abszesse, 5 mal 3 Abszesse. Von den 14 Abszessen saßen 5 im Kleinhirn, $4 \mathrm{im}$ Groß- und Kleinhirn, 4 in beiden Hirnhälften, 3 Abszesse wurden im Großhirn, and je einer im Kleinhirn und in beiden Großhirnhemisphären und in einem Kleinbirn gefunden.

In meiner Statistik habe ich 45 Fälle = circa 7 Proz. mit 2 Abszessen gefunden. Außerdem waren in einem Falle 3 Abs- 
zesse und 2 mal multiple Abszesse vorhanden. Die Fälle von 2 Abszessen verteilen sich folgendermaßen:

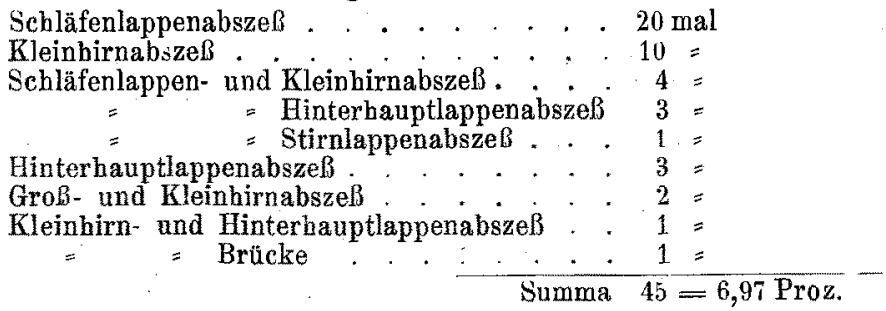

Wie schon seit längerer Zeit bekannt ist, ist der größte Teil der zur Entwicklung kommenden otitischen Hirnabszesse sehr nahe dem Orte des primären Leidens, oder in unmittelbarer Kontinuität mit denselben. Die von einem erkrankten Felsenbein ans induzierten Hirnabszesse liegen in demselben benachbarten Gehirnteilen, also im Schläfenlappen, seltener in anderen Teilen des Hirns. Diese Behauptung haben schon Mc Bride und Miller (19) im Jahre 1887 ausgesprochen. Etwas später als diese Autoren, (im Jahre 1889) ist dieselbe von Körner bestätigt worden. Nicht so ganz genan, aber in demselben Sinne ist dieser Satz schon von Toynbee aufgestellt worden. Er erklärt nämlich, daß Erkrankungen des äußeren Gehörganges sich auf das Kleinhirn verbreiten, der Pankenhöhle auf das Hirn, und des Labyrinthes auf den Bulbus. Nach seiner Meinung verbreitet sich die Entzündung der Warzenfortsatzzellen beim Kinde hauptsächlich auf das Hirn, beim Erwachsenen aber auf das Kleinhirn und auf den Sinus lateralis, was von verschiedener Beziehung dieses Knochens bei Kindern und Erwachsenen zum Centraluervensystem abhängig ist.

Es sind zwar in der älteren Literatur Fälle zitiert, wo der Abszeb auf der entgegengesetzten Seite der Ohreiterung sich befand, und man erklärte sich diese UnregelmäBigkeit durch „Fernwirkung", oder eine diskontinuierliche, sprungweise Verbreitung der parasitären Mikroorganismen, es scheint aber, daß solche Fälle auf diagnostischen Irrtümern beruhen. Ioh glaube, daß man es in solchen Fällen mit keinem wahren otitischen Abszeß zu tun hatte, oder es wurde der primäre Krankheitsherd im Ohre iubersehen. In meiner Statistik fand ich im ganzen zwei Fälle, wo der Abszeb nicht auf derselben Seite wie die Obreitcrung sich befand, und zwar einmal bei Hansberg (Nr. 203) und einmal bei R. Müller (Nr. 412), referiert von Grunert. -- Teh vermute, daß in diesen beiden Fällen nur ein Lapsus calami vor- 
handen ist. Nach meiner Erfahrung sind und können die wahren otitischen Abszesse nur auf der Seite des erkrankten Ohres sich befinden. Erkrankungen im Bereiche der mittleren Schädelgrube führen somit zu Abszessen im Schläfenlappen, selten zu Abszessen der übrigen Großhirnteile, solche im Gebiete der hinteren Schädelgrube zum Abszesse im Kleinhirn. Körner sammelte im ganzen acht Fälle von Großhirnabszessen bei Knochenerkrankung in der Kleinhirngrube (Fälle von Jacoby, Ollivier, Bezold, Hansberg, Schmiegelow, Morf, Gradenigo, Martin-Meyer) und einen Fall von Kleinhirnabszeh bei Erkrankung der mittleren Schädelgrube (Fall von Dupuy). In der großen Mebrzahl der Fälle werden die otitischen Hirnabszesse nicht durch Sebleim hauterkrankungen in den Hohlräumen des Sehläfenbeins, sondern durch Krankheiten des Knochens selbst hervorgerufen.

Um das Verhältnis der Komplikationen, die den otitischen Hirnabszeß begleiten oder durch ibn hervorgerufen werden, festzustellen, habe ich die Zahl und Art der Komplikationen bei geheilten und tödlichen, akuten und chronischen Fällen gesammelt. Im ganzen babe ich auf 645 Fälle 309 mal Komplikationen, also fast in der Hälfte der Fälle, konstatiert. - Die Komplikationen sind in folgender Tabelle dargestellt:

\section{Komplikation}

Sinusthrombose

Sinusthromb. u. extradur. Abszeß

Extraduraler Abszeß

Extradural Abszeß u. Man:

Pachymeningitis

Encephalomeningitis $\cdot 2$

Pneumonie . . : : $:=-$

Ruptura a. carotis interna

Summa 11
Geheilte Fälle Tödliche Fälle akute chron. akute chron.
Char. d. Ohrenl. n. angeg.

Aus dieser Tabelle ergibt sich, daß die öfteste tödliche Komplikation der otitischen Hirnabszesse die Meningitis ist, den zweiten Platz nimmt die Sinusthrombose ein. Bei den greheilten Fällen wird am häufigsten der extradurale Abszeß und die Pachymeningitis beobachtet. Dieselbe Tabelle führt aber auch zur Vermutung, daf otitische Hirnabszesse, die mit anderen schweren Komplikationen verbunden sind, gewöhnlich tödlich verlaufen. Hirnabszesse mit minder schweren Komplikationen müssen aber auch prognostisch für sehwer betrachtet werden. Aus meiner Statistik ist vielleicht der Schluß zu ziehen, daß die otitischen Hirnabszesse in ihren früheren Perioden wahrseheinlich von min- 
der schweren Komplikationen, als in ihrem weiteren Verlaufe begleitet sind. Sollte dem wirklich so sein, wäre dies ein ungemein wichtiger Wink für die Therapie.

Hessler fand auf 67 reine Großhirnabszesse 26 die mit Meningitis und 13 die mit Sinusthrombose kompliciert waren. Auf 43 reine Kleinhirnabszesse waren 6 mit Meningitis und 10 mit Sinusthrombose komplieiert. Anf 9 Grob- und Kleinhirnabszesse war 1 mal Meningitis und 2 mal Sinustbrombose vorhanden. Im allgemeinen hat Hessler bei 119 Hirnabszessen $33 \mathrm{mal}$ die Meningitis und $25 \mathrm{mal}$ die Sinusthrombose gefunden.

Röpke sammelte auf 142 operierte Fälle 80 Todesfälle, bei denen folgende Komplikationen beobachtet wurden:

Der Tod erfolgte durch Unglücksfälle, die mit der Operation in

Zusammenhang waren

Durchbruch in den Seitenventrikel vor der Operation : $5=$

Durchbruch in den Seitenventrikel nach der Operation mit Komplikation

Meningitis vor der Operation nach der Operation

Encephalomeningitis

Zweiter uneröffneter Abszeß

Sinusphlebitis and Pyaemie

und Meningitis

Himprolaps-Meningitis

Nene Abszesse

Apoplexie

Preumonie

Unterleibserkrankung

Die Todesursache nicht erwähnt

Keine Antopsie und Encephalitis

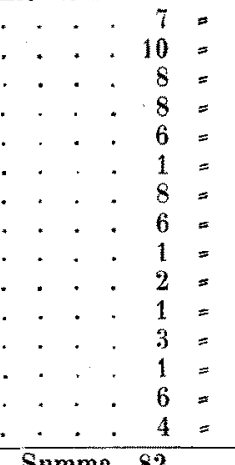

Hessler und Röpke fanden ebenso wie ich, daß die Meningitis die öfteste Komplikation der otitischen Hirnabszesse ist.

Das gegenseitige Verhältnis der otitischen Hirnabszesse zu anderen letalen Komplikationen der Ohreiterungen entnehme ich teilweise aus meiner früheren Arbeit (20), dasselbe stellt sich folgendermaßen dar:

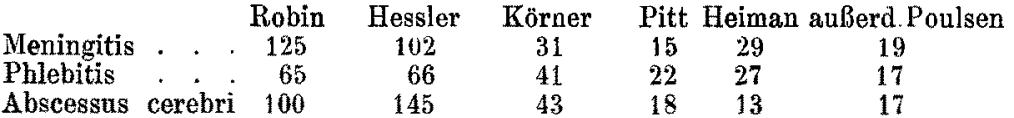

Gruber fand bei Zählung der unkomplicierten Fälle folgendes Verhältnis:

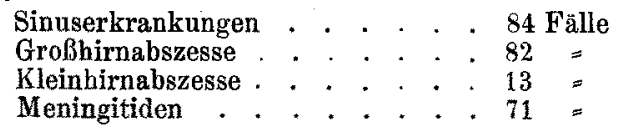

Faßt man die Ziffer aller dieser Statistiken zusammen, bekommt man: Meningitis 392 mal, Hirnabszeß 381 mal und Sinus- 
erkrankung 322 mal. Also das Übergewieht der Meningitis über andere Komplikationen der Ohreiterungen. -

Otitische Hirnabszesse entwickeln sich sehr häufig bei chronischen Mittelohreiterungen. Früher wurden sie bei akuten Obreiterungen für selten betrachtet. Nach einer von Grunert(21) aus der Literatur zusammengestellten Statistik ist die den Abszeß indueierende Ohreiterung in 91 Proz. der Fälle ehronisch und in 9 Proz. akut. Koch fand auf 85 chronische Fälle 15 akute. Bei Hammerschlag sind nur schon 75 Proz. der Fälle nach chronischen Ohreiterungen und 25 Proz. nach akuten. Ich fand 457 chronische, 113 akute Fälle, und $75 \mathrm{mal}$ war der Charakter der Ohreiterung unbekannt. In meiner Statistik ist das Verbältnis der nach ebronisohen Ohreiterungen zu den nach akut entstandenen Hirnabszessen 80,17 Proz.:19,83 Proz. Okada ist auch der Meinung, daß Kleinhirnabszesse nach akuten Obreiterungen nicht selten sind, unter seinen sämtlichen Fällen fand er dieselben in 17 Proz. Er glaubt, daß die Entstehung der Kleinhirnabszesse bei akuten Obreiterungen vom Alter der Kranken abhängig sei. Unter 28 Fällen fand er sie im ersten Decennium 6, im zweiten 5 , im dritten 13 und in den weiteren Lebensjahren 4 mal.

Was die Akuität und Chronicität der otitischen Hirnabszesse betrifft, ist $\mathrm{Körner}$ der Meinung, daß sie in der Regel chronisch sind und seit einer langen Reihe von Jahren bestehen. Ma cew en nimmt anf Grund der publicierten Fälle an, daß nur der kleinere Teil der otitisehen Hirnabszesse als chronisch bezeichnet werden darf. Dies beweist anch die Kasuistik von Röpke. Von 142 operierten Großhirnabszessen sind vom Eintritt der ersten Symptome ab gerechnet 58 Fälle akuter, 16 subaknter und nur 11 chronischer Natur gewesen; bei 57 Fällen fehlen Angaben aus denen man auf den Verlauf des Abszesses einen Schluß ziehen könnte. - Ich habe diese Frage bei der Zusammenstellung meiner Statistik außer Acht gelassen denn ist es für die Prognose und Therapie gleichgültig, ob der Abszeß akut oder chronisch ist; außerdem sind wir in den meisten Fällen nicht imstande, die Anfangsperiode des Abszesses zu bestimmen und das hauptsächlich bei chronisehen Ohreiterungen. Mustert man genau die Kasuistik der veröffentlichten otitisehen Hirnabszesse durch, so tiberzeugt man sich, daß der größte Teil derselben von nicht langer Dawer ist, eventuell nicht lange her der Verdacht auf einen Abszeß entstand. Man könnte dagegen einwenden. daß die klinische Diagnose der anatomischen nicht entspricht. Die Erfahrung lehrt 
aber, daß Hirnabszesse ohne klinische Symptome zu den Seltenbeiten gehören.

Über die Operations- eventuell Heilresultate bei otitischen Hirnabszessen berichtet $\mathrm{K}$ örner in den verschiedenen Ausgaben seines Werkes ïber folgende Ziffern:

$$
\begin{aligned}
& \text { Jahr } 189346 \text { operierte Grobhirnabszesse mit } 56,5 \text { Proz. Heilungen } \\
& \text { und } 9 \text { Kleinhimabszesse mit } 33,3 \text { Proz. Heilungen } \\
& \text { Jahr } 189676 \text { operierte Großhirnabszesse mit 55,5 Proz. Heilungen } \\
& \text { und } 16 \text { Kleinhirnabszesse mit 56,25 Proz, Heilungen } \\
& \text { Jahr } 190 \mathrm{I} 212 \text { operierte Großhirnabszesse mit 50,5 Proz. Heilungen }
\end{aligned}
$$

Körner fand bis Oktober 1901267 HirnabszeBeröffnungen mit 137 Heilungen, abgesehen von zahlreichen vergeblichen, meist lediglich zur Exploration vorgenommenen Operationen.

Toh habe vor mehreren Jahren (22) 32 Falle operierter otitischer Hirnabszesse aus der Literatur gesammelt, darunter waren 17 Heilungen.

Schwartze und Broca-Mauriac schätzen in ihren Werken die Zahl der duroh Operation geheilten Fälle von otitischen Hirnabszessen auf 00 Proz. 0 p penh eim fand auf 104 operierte Großhirnabszesse 48 Heilungen, was 46,1 Proz. ausmacht. Nach Röpke, der, wie schon oben erwähnt wurde, 142 operierte Fälle sammelte, waren 59 Heilungen $=48,4$ Proz. Von den duroh akute Obreiterung inducierten 26 Abszessen wurden $11=42,3$ Proz. geheilt, von den infolge shronischer Otorrhoe entstandenen $109 \mathrm{Abs}-$ zessen wurden $47=43,1$ Proz. geheilt. Nach Röpke ist es für die Prognose der Hirnabszeßoperationen ziemlich gleich, ob das primäre Leiden akuter oder chronischer Natur ist. Dagegen bieten die akut oder subakut verlaufenden Prozesse eine bedeutend günstigere Chance für die Heilung, als die ohronischen, denn von

58 akut verlaufenden Fällen wurden geheilt $30=51,7$ Pro

16 subakut $=\quad=8=50=$

11 chronisch $=\quad=\quad=2=18,1=$

Hammerschlag sammelte 53 Fälle operierter otitischer Hirnabszesse, davon wurden geheilt $26=49,05$ Proz.

$0 \mathrm{k}$ a d a (23) erhielt 16 Heilungen auf 43 Fälle von Kleinhirnabszessen,was 37,21 Proz. ausmacht. Unter den 27 tödlichenFällen waren 18, in denen der Abszeß erst bei der Auto psie entdeekt wurde.

Aus meiner Statistik, die 645 enthält, nach Ausscheidung von 109 Fällen, in denen die Operation nicht ausgeführt wurde, wie auch 17, wo nur die Mastoidoperation gemacht wurde, wurde 519 mal die Abszeßentleerung ausgeübt. Die Heilungsresultate waren folgende: 


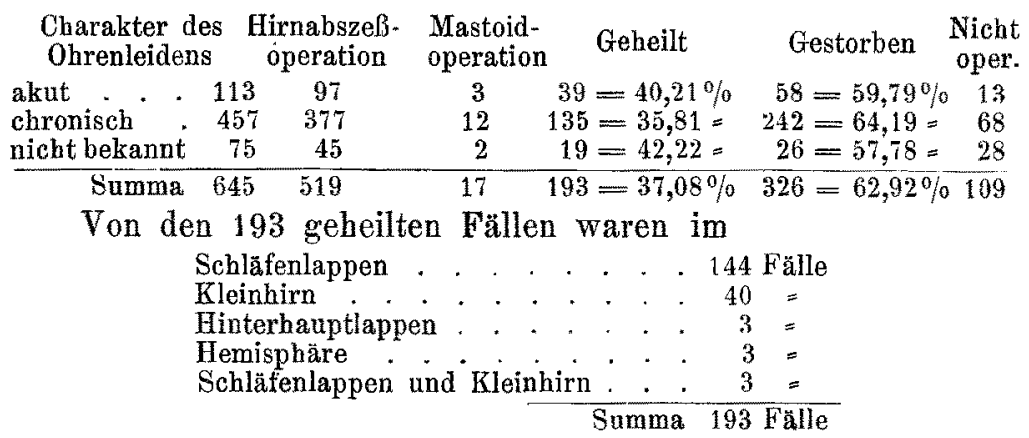

Das Verhältnis der geheilten Großhirnabszesse zu den geheilten Kleinbirnabszessen stellt sich heraus 79,27:20,73 Proz. In 20 von den operierten Abszessen wurde derselbe erst bei der Autopsie gefunden. Dreimal wurde derselbe im Großhirn gesucht und im Kleinhirn gefunden.

Die Resultate meiner Statistik bew eisen, dab in Fällen, wo der HirnabszeB durch akute Ohreiterung indueiert war, die Heilungsresultate etwas besser sind, als da, wo die Ursache des Abszesses eine chronische Mittelohreiterung war. Im ersten Fall ist der Prozentsatz höher als 40, im zweiten erreicht er nicht dieZiffer 36. Der Prozentsatz der Heilungen der Großhirnabszesse ist fast viermal so groß als der der Kleinhirnabszesse - ein recht wichtiges Moment für die Prognose der otitischen Hirnabszesse. Die Heilungsziffer der Grob- und Kleinhirnabszesse in meiner Statistik variiert stark von der Heilungsziffer aller anderen Beobachter, wo dieselbe für Grob- und Kleinhirnabszesse recht gleich ist.

Ich betrachte die in meiner Statistik ausgedrückten Zifferm der Heilungen nach Hirnabszeßoperationen für noch zu hoch und der Wahrheit nicht entsprechend, und zwar deshalb, daß eine ganze Reihe von mißglückten Fällen nicht veröffentlicht wird. Meinen Scbluß ziehe ich aus folgendem Umstand: Vergleicht man die Heilungsresultate mancher größerer Kliniken, wo kein Fall verheimlioht wird, so überzeugt man sich, daß da die Heilungsziffer viel niedriger unter der von mir angegebenen steht. Unter 41 operierten Fällen aus der Hallenser Klinik fand ich im ganzen 8 geheilte Fälle (siehe Tabelle I) bei 34 Todesfällen, also 17,07 Proz. Heilungen. Von diesen 7 geheilten Fällen ist der Abszeb zweimal bei akuter Mittelohreiterung und fünfmal bei ehronischer entstanden. Preysing citiert 10 Fälle operierter Hirnabszesse, darunter waren $3=30$ Proz. Heilungen und $7=70$ Proz. Todesfälle. Tenzer gibt an 17 operierte chronische Fälle aus der 
Berliner Charité, und da finden wir 11 Todesfälle (1 nnoperierter Fall) und 6 Heilungen, was 37,5 Proz. ausmacht. R. M $u ̈ 1 l \mathrm{er}$ citiert aus der Trautman n schen Klinik 9 Fälle, darunter 3 Heilungen und 6 Todesfälle. $0 \mathrm{kada}$ operierte $3 \mathrm{mal}$ mit ungünstigem Ausgang, ebenso Jansen 3 mal, Ich habe auf 8 operierte Fälle 2 Heilungen erhalten. Vergleicht man diese Ziffern mit denen anderer Antoren, wie z. B. Heine, Man a s se usw., die keine tödlichen Fälle verzeichnen, so wirft sich unwillkürlich der Gedanke auf, daß diese Autoren nur die günstigen Fälle veröffentlichten, während sie die ungünstigen unberücksichtigt ließen. Macewen ist der einzige Operateur, der sehr günstige Resultate erreicht hatte, ca. 80 Proz.

Wenn also solch ausgezeichnete Kliniken, wie die Hallenser und andere von mir citierte Austalten, wo alle wissenschaftlichen und technischen Bedingungen mit der größten Sorgfalt ausgeführt werden, nicht einen sehr glänzenden Prozentsatz von Heilungen nach otitischen HirnabszeBoperationen aufzuweisen haben, mutssen wir heutzutage hohe Ziffern unbedingt für unrichtig halten. Nach den bisherigen Erfahrungen müssen 40 Proz. Heilungen als die böchste Ziffer betrachtet werden. Zu Ungunsten der Heilresultate spricht der Umstand, daß mehrere als geheilt publicierte Fälle zur Zeit der Publikation noch nicht lange genug beobachtet waren, um als "dauernd" geheilt bezeichnet zu werden. In den von mir gesammelten Fällen ist z. B. ein Fall angegeben, der schon 4 Tage nach der Operation als geheilt angegeben wird. Es sind doch auch die üblen Folgezustände zu berücksichtigen, die nicht selten nach der vermeintlichen Heilung eintreten. Namentlich hat Schwartze verschiedentlich über diese Tatsachen seinem Bedanern Ausdruck gegeben. Auch Körner erwähnt, daß nur von wenigen Operierten Angaben vorliegen, dab sie mehr als naeb einem Jahre noch lebten. Die Kasuistik liefert zwar von Jahr zu Jahr bessere Operationsresultate, nieht destoweniger halte ich den heutzutage angegebenen Prozentsatz der geheilten Fälle fur zu hoch, und wie bis jetzt, beharre ich auf den Standpunkt, den ich in meiner Arbeit (24) ausgesprochen habe. -

Von einer Statistik uber spontan gebeilte Fälle von otitisehen Hirnabszessen kann nieht die Rede sein. In meiner Statistik ist ein einziger solcher Fall, den Brieger beobachtete, erwähnt. Spontane Heilungen haben Broussais (1824), viel später Braun nnd Sutphen beobachtet. 


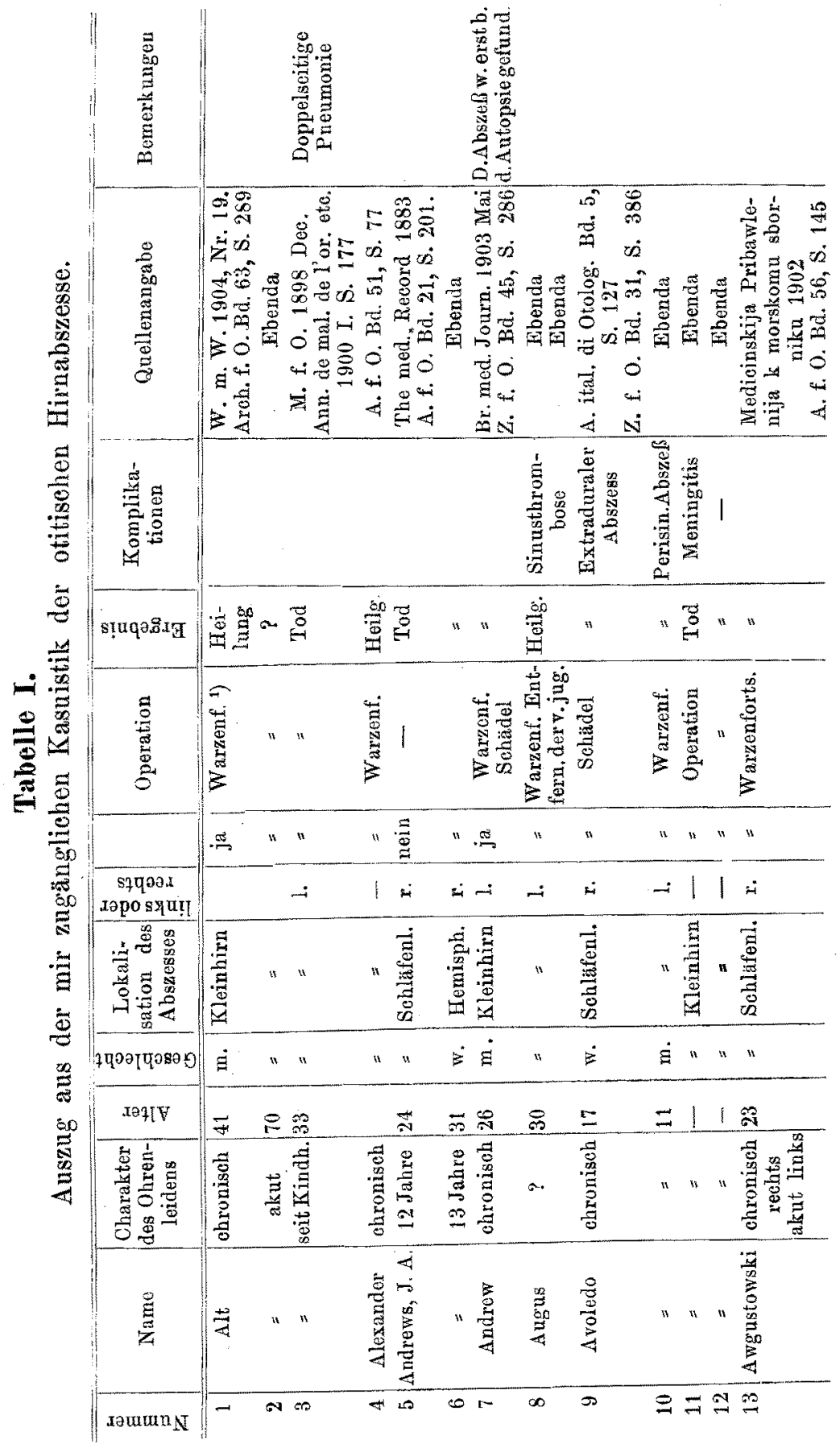




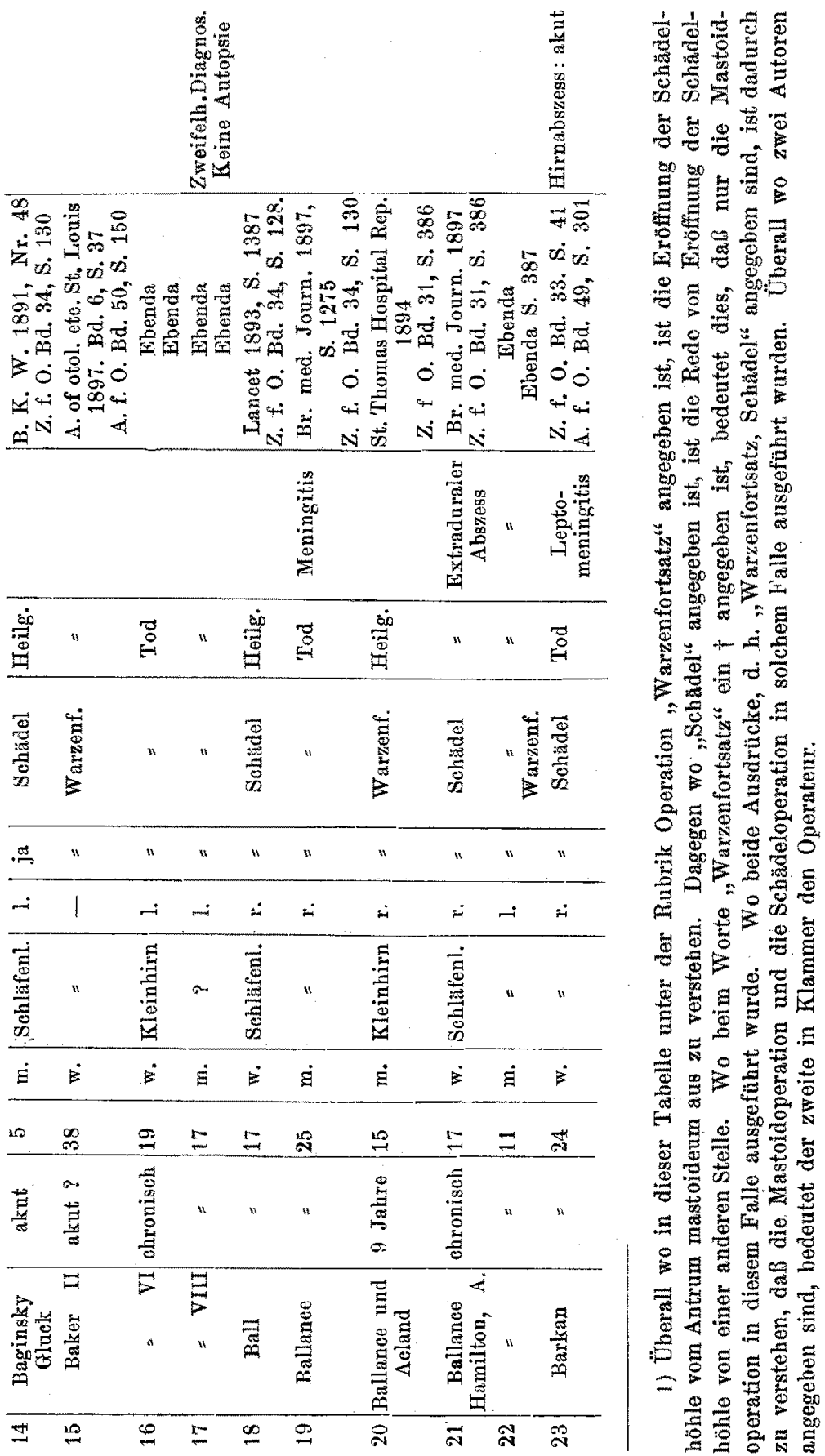




\begin{tabular}{|c|c|c|c|c|c|c|c|c|c|c|}
\hline 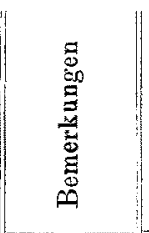 & 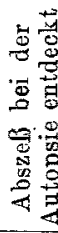 & 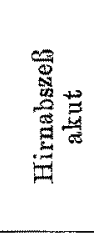 & & 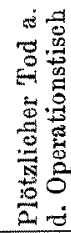 & & & 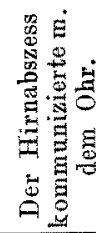 & & & 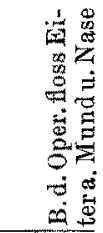 \\
\hline 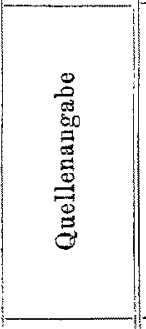 & 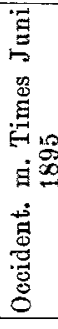 & 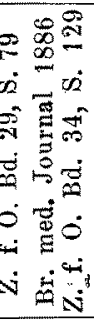 & & 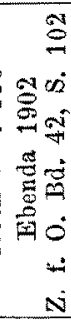 & 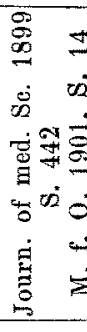 & 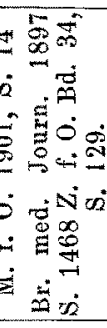 & 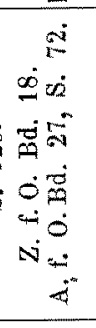 & 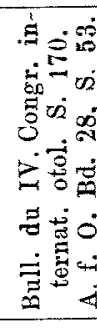 & 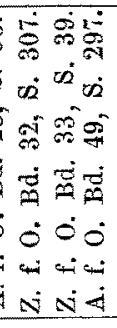 & 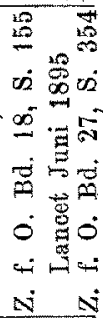 \\
\hline 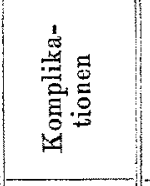 & & & & & & & & & 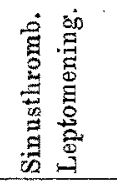 & \\
\hline sṭuqə.̆.xt & $\stackrel{\overrightarrow{0}}{\leftrightarrow}$ & $\begin{array}{l}\text { 蛋 } \\
\end{array}$ & $"$ & $\stackrel{\mathrm{g}}{\mathrm{g}}$ & " & 高 & $"$ & $"$ & $\vec{D}_{0}$ & 焉 \\
\hline \multirow[t]{2}{*}{ 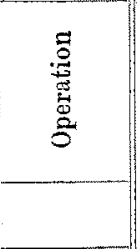 } & 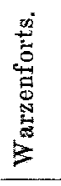 & 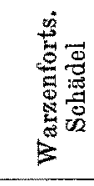 & " & 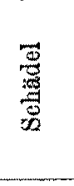 & 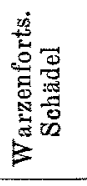 & $"$ & 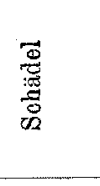 & 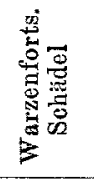 & 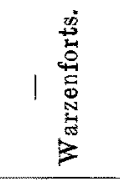 & 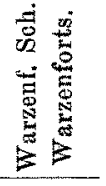 \\
\hline &.$\stackrel{s}{.}$ & $"$ & $"$ & $"$ & " & $"$ & " & $"$ & 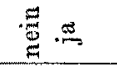 & " " \\
\hline 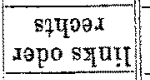 & - & $\dot{4}$ & $\dot{-1}$ & & s. & $\dot{-1}$ & $\therefore$ & $\stackrel{\dot{ }}{-}$ & $\dot{4} \rightarrow$ & $\dot{\mu} \alpha$ \\
\hline 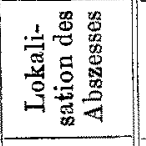 & 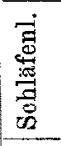 & $"$ & $"$ & 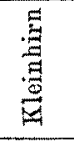 & 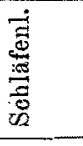 & $"$ & $"$ & $"$ & 烝" & 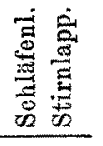 \\
\hline 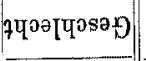 & $\dot{\xi}$ & 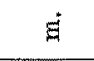 & $"$ & $\dot{E}$ & $\sim$ & $\dot{B}$ & $\dot{\varepsilon}$ & $"$ & " " & an. \\
\hline xəキโष & 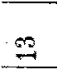 & 2 & 鄵 & \pm & $\infty$ & 8 & $\frac{\text { sa }}{3}$ & $\bar{a}$ & $2:=$ & 0. \\
\hline 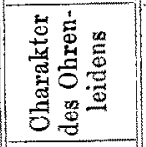 & $\overrightarrow{\vec{z}}$ & 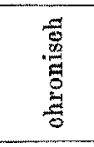 & " & " & $\overrightarrow{\vec{Z}}$ & 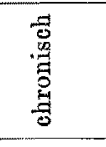 & ․ㅡㄹ & 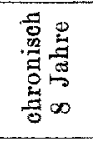 & 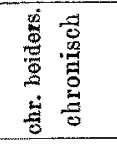 & " \\
\hline 尝 & 番 & 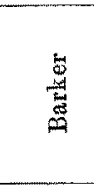 & " & " & 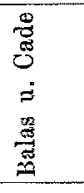 & 胥 & 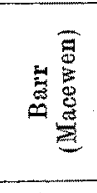 & 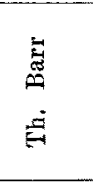 & " " & 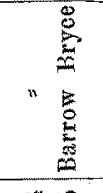 \\
\hline $\mathbf{N}$ & $\bar{N}$ & $\stackrel{L}{\circ 9}$ & $\ddot{\text { ส }}$ & $\mathrm{N}$ & $\stackrel{\infty}{\sim}$ & $\stackrel{8}{8}$ & 요 & $\vec{\infty}$ & 粗 & 范哭 \\
\hline
\end{tabular}




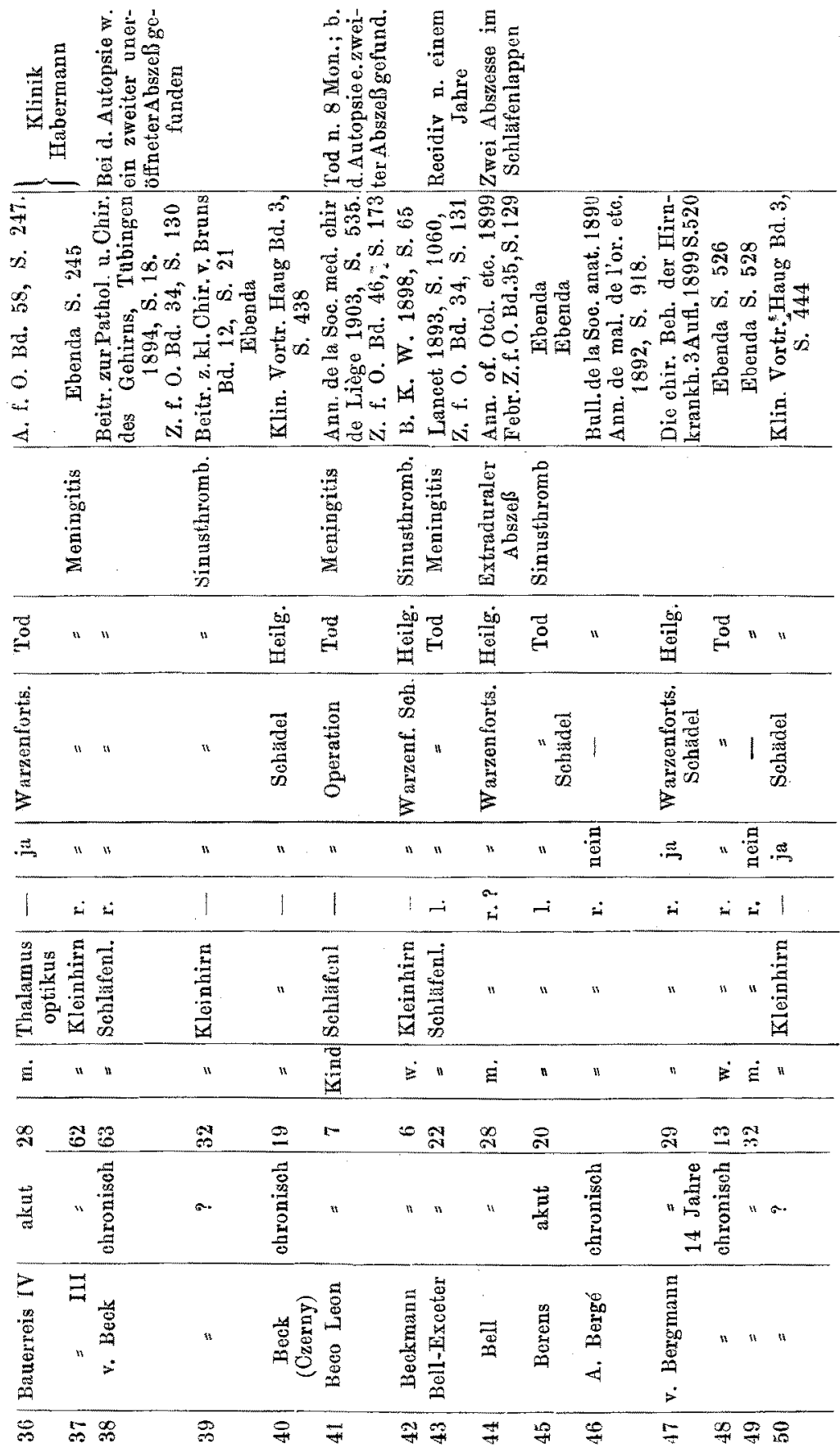




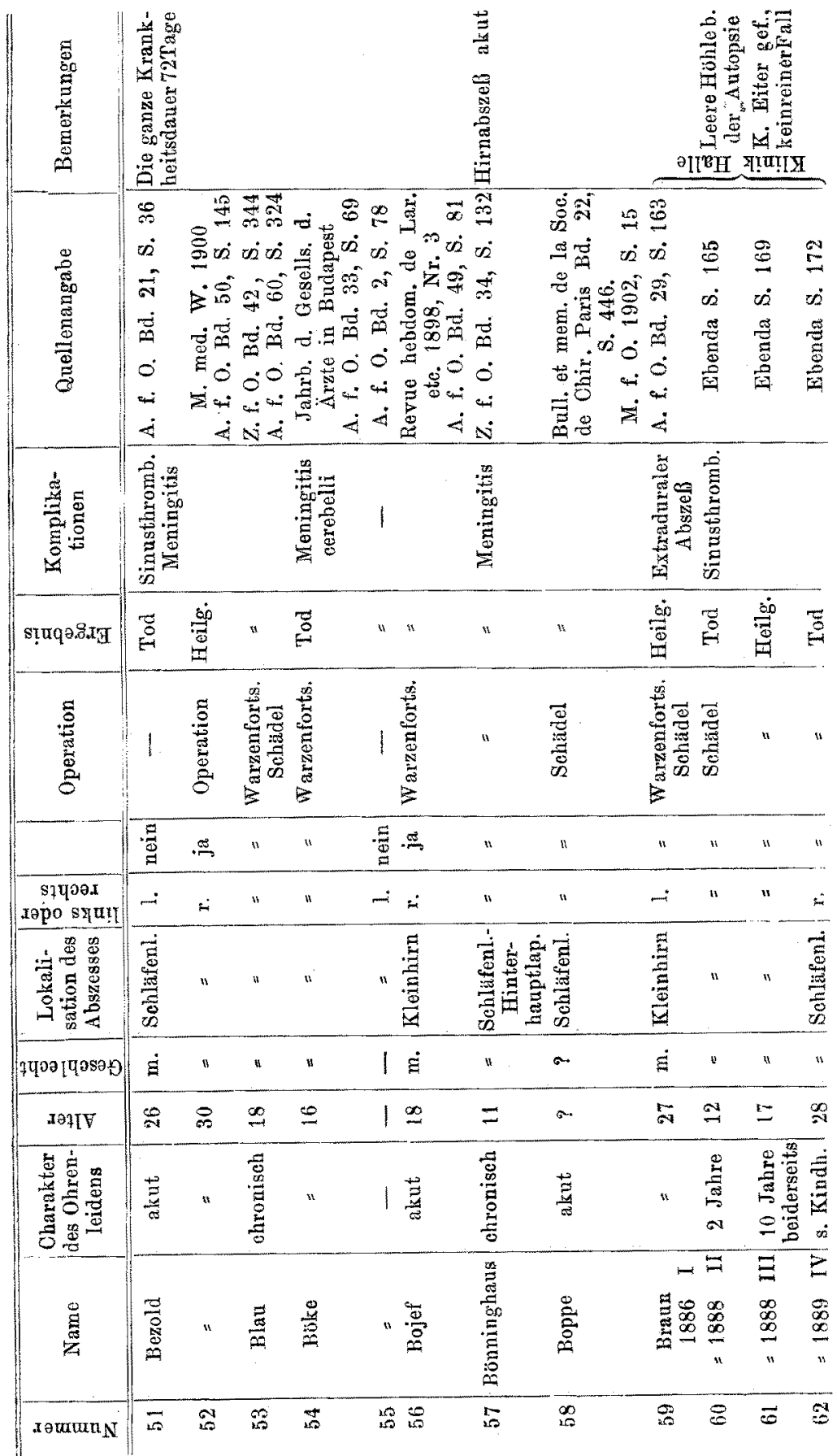




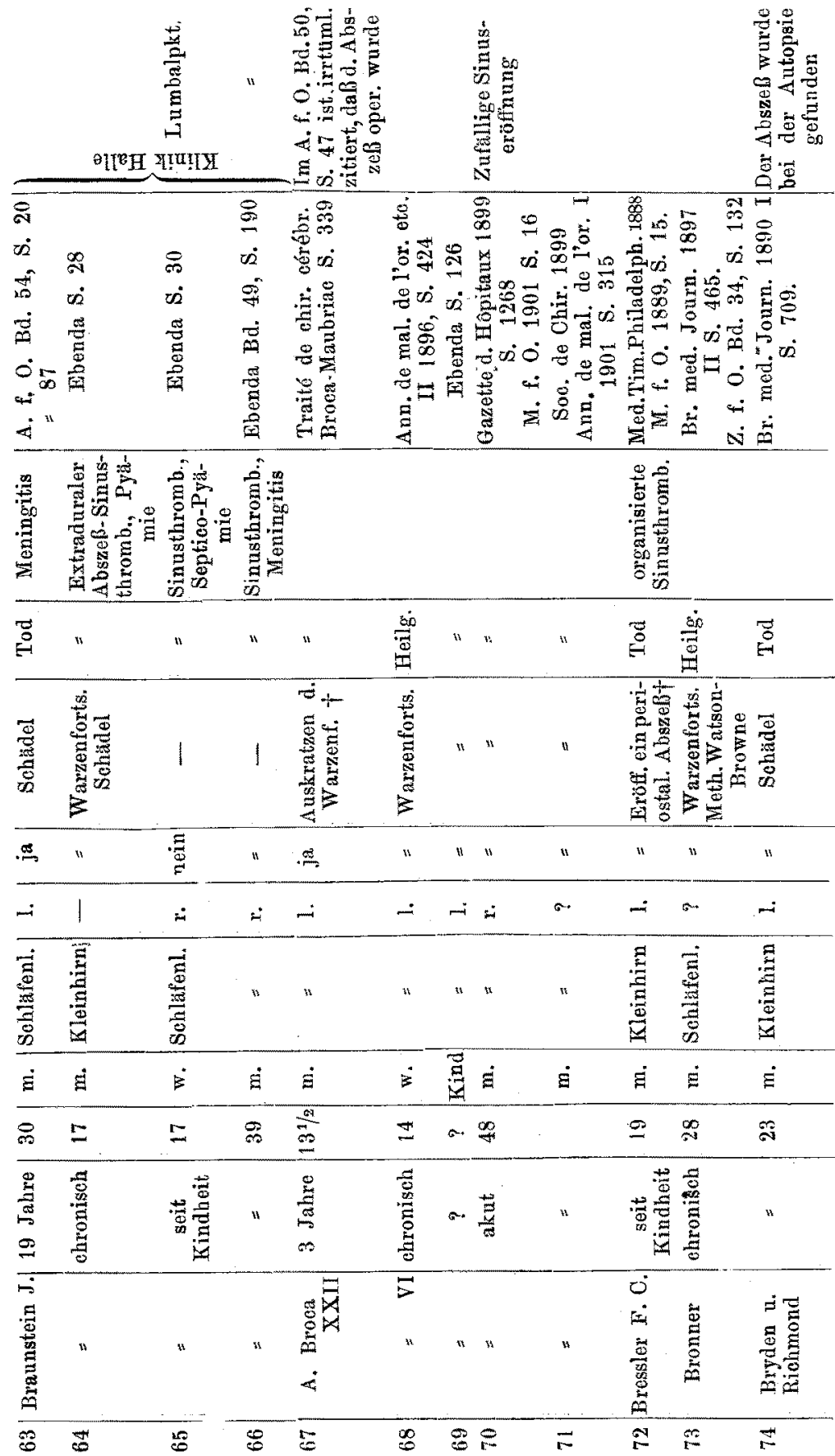




\begin{tabular}{|c|c|c|c|c|c|c|c|c|c|}
\hline 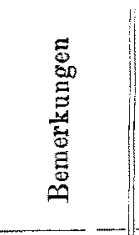 & & & 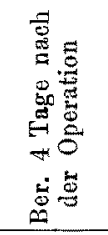 & & & 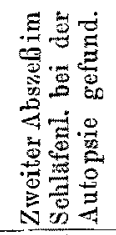 & & & 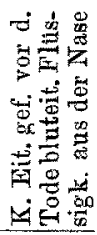 \\
\hline 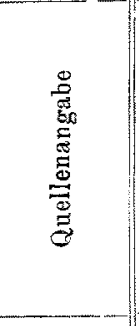 & 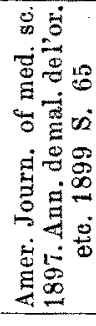 & 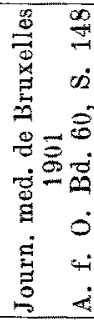 & 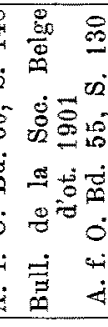 & 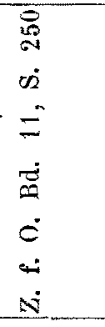 & 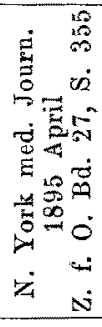 & 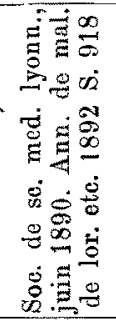 & 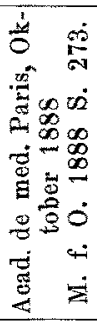 & 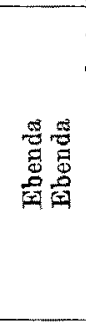 & 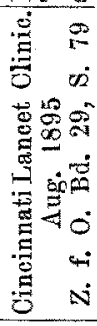 \\
\hline 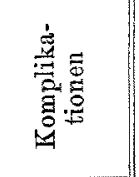 & 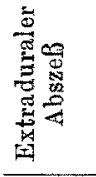 & w & & 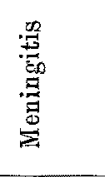 & & & 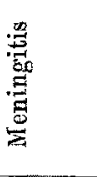 & 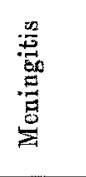 & \\
\hline s!uq & $\begin{array}{r}\vec{D} \\
E-1\end{array}$ & 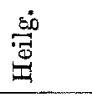 & $\approx$ & $\ddot{8}$ & $"$ & $"$ & $"$ & n & n \\
\hline 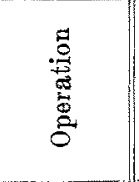 & 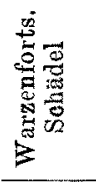 & n & " & 1 & 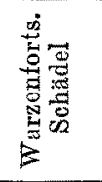 & 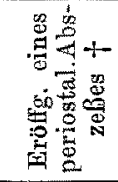 & 1 & $\underset{5}{+}$ & 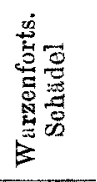 \\
\hline & 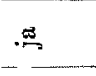 & $n$ & " & $\Xi$ &. & $"$ & $\stackrel{\Xi}{g}$ & .0 & $"$ \\
\hline 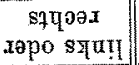 & - & - & - & & & $\dot{2}$ & $\dot{-}$ & 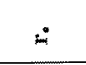 & - \\
\hline 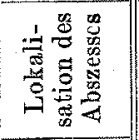 & 总 & " & " & 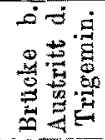 & 急 & $n$ & $\begin{array}{l}\frac{\vec{g}}{9} \\
\frac{3}{3} \\
\frac{3}{8} \\
0\end{array}$ & " & s. \\
\hline 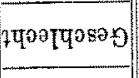 & $\dot{\Xi}$ & $\begin{array}{l}3 \\
\end{array}$ & घं & " & $"$ & $"$ & $"$ & " & " \\
\hline .əجโพ & ஜ & $\exists$ & $\Longrightarrow$ & $=$ & $\stackrel{2}{\rightarrow}$ & 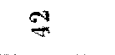 & $\approx$ & & $\infty$ \\
\hline 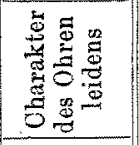 & 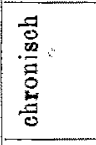 & $"$ & $"$ & 采 & 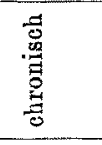 & 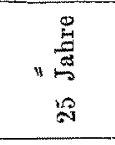 & 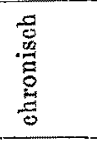 & 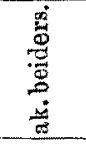 & $\frac{\overrightarrow{\vec{G}}}{\vec{\Xi}}$ \\
\hline 莺 & 莣 & 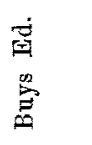 & 窟 & 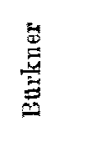 & 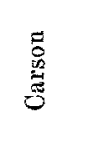 & 晃 & $\underset{0}{0}$ & " & 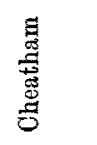 \\
\hline *ə rumn $N$ & 10 & $\varphi$ & $E$ & $\infty$ & $R$ & 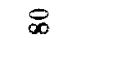 & $\vec{D}$ & $\propto$ & $\mathscr{\infty}$ \\
\hline
\end{tabular}




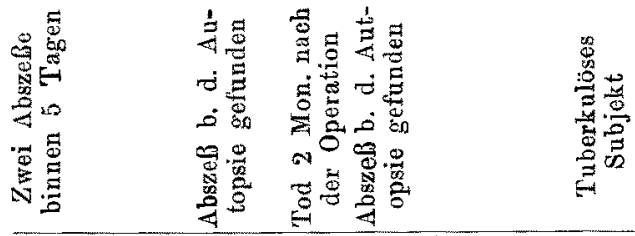

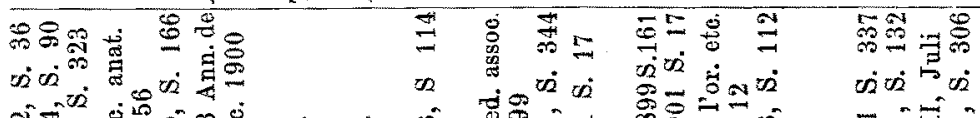
जी

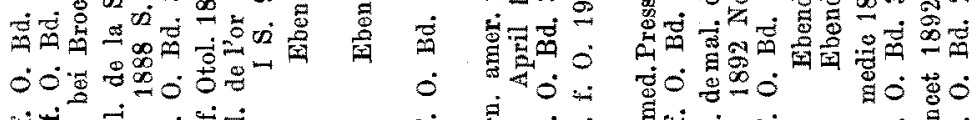

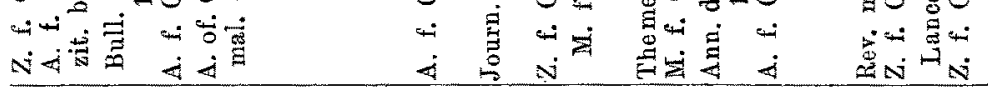

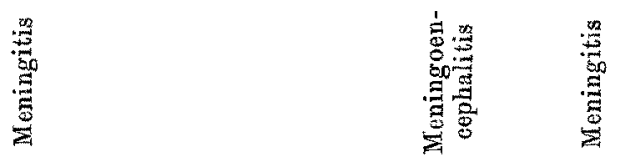

\begin{tabular}{|c|c|c|c|c|c|c|c|c|c|c|c|c|}
\hline$\stackrel{5}{5}$ & $\begin{array}{l}8 \\
0 \\
0\end{array}$ & " & " & " & " & " & " & $\stackrel{80}{\overrightarrow{8}}$ & $\begin{array}{l}\vec{\circ} \\
\stackrel{0}{1}\end{array}$ & " & " & $\frac{8}{3}$ \\
\hline 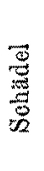 & 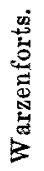 & 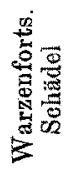 & $\frac{\frac{n}{3}}{\frac{d}{2}}$ & 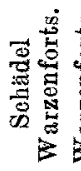 & 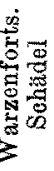 & 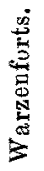 & " & 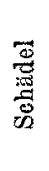 & 1 & 1 & 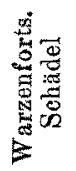 & " \\
\hline 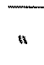 & $n$ & n & $n$ & 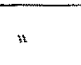 & $n$ & a & 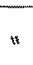 & n & 要 & n &. & " \\
\hline
\end{tabular}

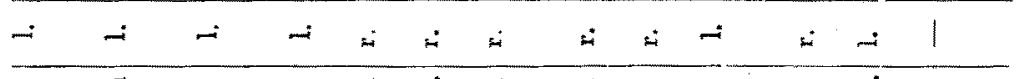

\begin{tabular}{|c|c|c|c|c|c|c|c|c|c|c|c|}
\hline 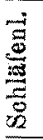 & 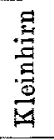 & 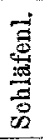 & 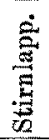 & 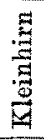 & 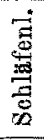 & 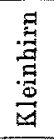 & 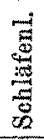 & $"$ & $"$ & 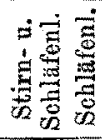 & 吾 \\
\hline घ̈ & $"$ & $n$ & 0 & $\sum^{*}$ & $\dot{घ}$ & $"$ & : & 7 & $F$ & $n$ & $\sum^{\circ}$ \\
\hline
\end{tabular}

\begin{tabular}{|c|c|c|c|c|c|c|c|c|c|c|c|c|}
\hline ज्ञ & $\Xi$ & & 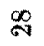 & $\stackrel{\infty}{\sim}$ & $\mathscr{8}$ & $\cong$ & $\underset{\sim}{\infty}$ & స્心 & $\stackrel{\Re}{\sim}$ & $\vec{\sigma}$ & 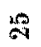 & \pm \\
\hline 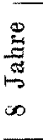 & 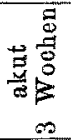 & 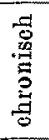 & $\begin{array}{l}\stackrel{ \pm}{\vec{z}} \\
\stackrel{y}{*}\end{array}$ & " & & & " & " & $"$ & " & " & 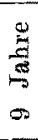 \\
\hline
\end{tabular}

\begin{tabular}{|c|c|c|c|c|c|c|c|c|c|}
\hline 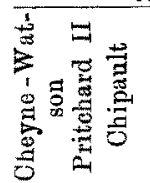 & $\stackrel{8}{8}$ & " & " & 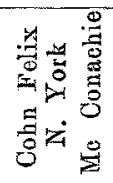 & 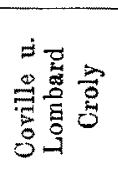 & 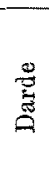 & " & 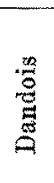 & $\stackrel{\Xi}{\Phi}$ \\
\hline$\infty$ & $\infty$ & $\infty$ & $\infty$ & 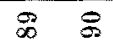 & $\bar{\sigma}$ & 8 & $\sigma$ & 8 & $\%$ \\
\hline
\end{tabular}




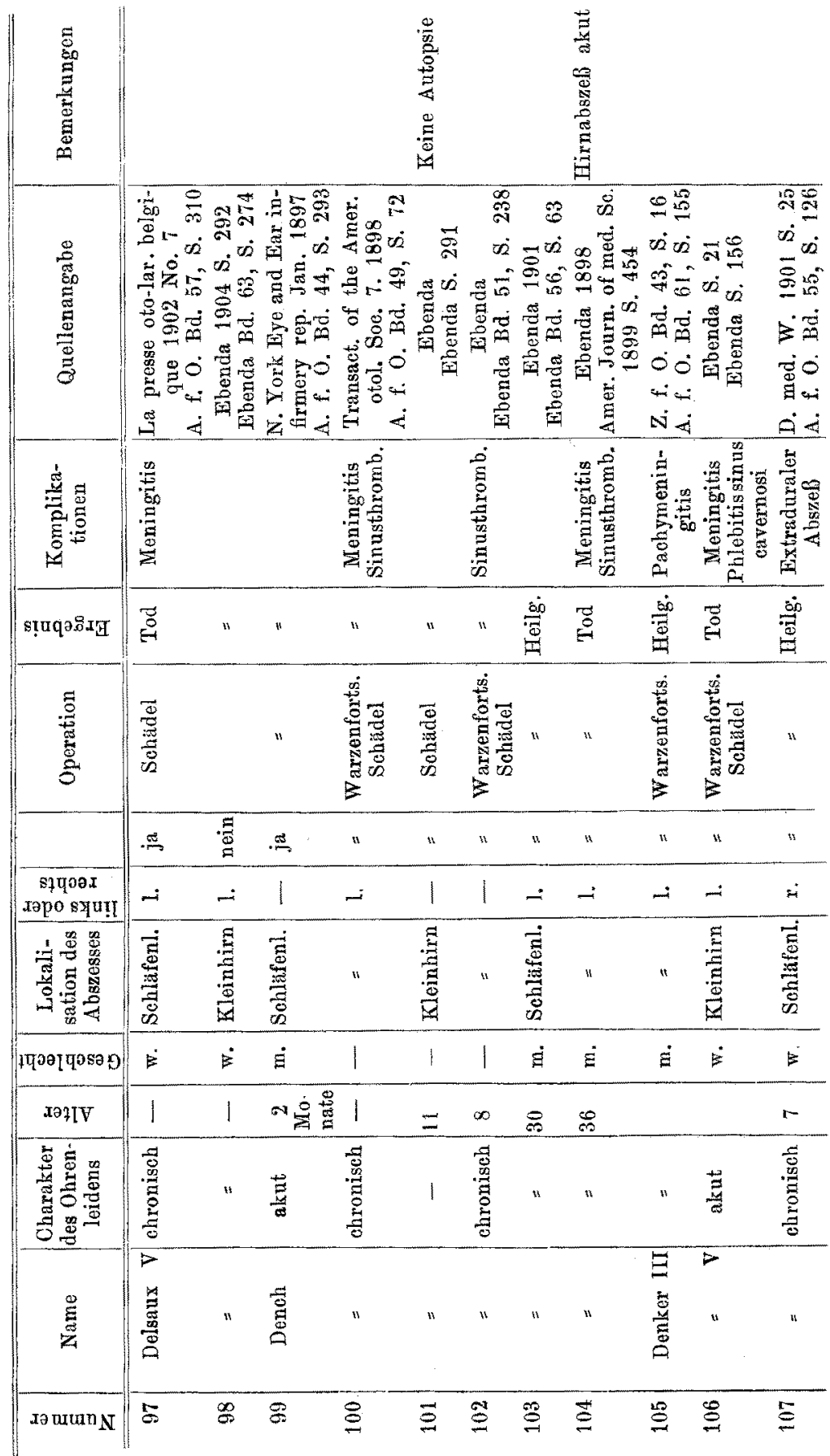


Ein Fall von akutem otitischen Schläfenlappenabszeß.

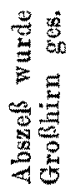

a.
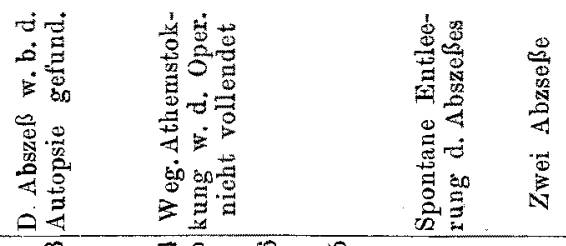

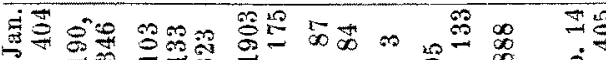

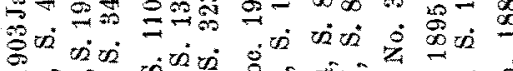

或地 की

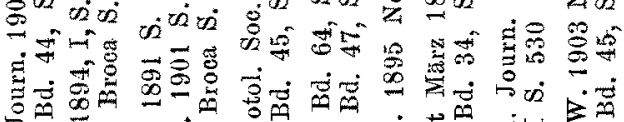

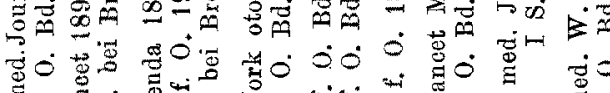

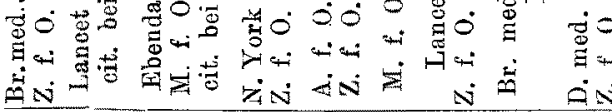

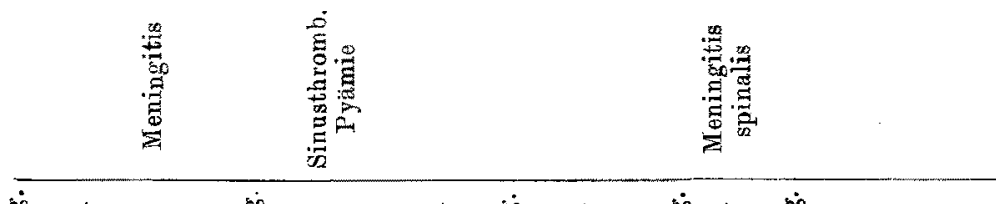

i

on

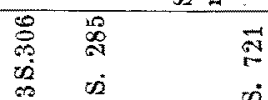

$\overrightarrow{\mathrm{N}}$

का को को

$\dot{0}$

总总悉

"

i

$\dot{0}$

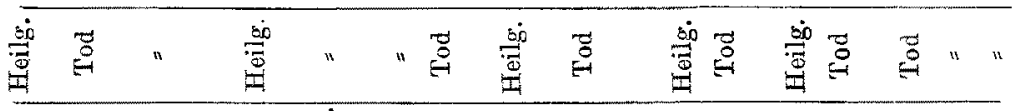

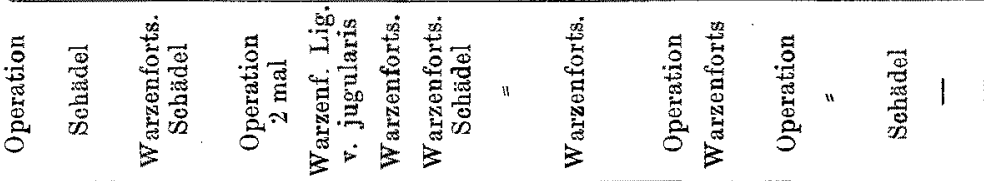

\begin{tabular}{|c|c|c|c|c|c|c|c|c|c|c|c|c|}
\hline & " & " & " & " & " & " & $"$ & " & " & $"$ & " & " 若 \\
\hline & $\dot{H}$ & 1 & - & - & $\dot{\sim}$ & - & $-i$ & 1 & $-i$ & $\dot{H}$ & $=$ & $\rightarrow$ \\
\hline
\end{tabular}

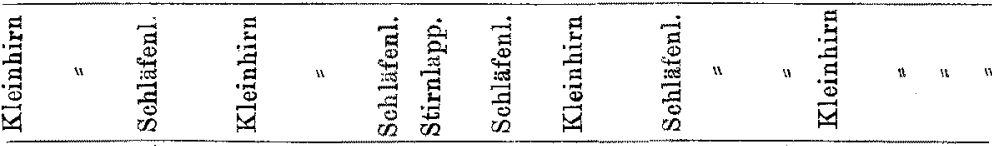

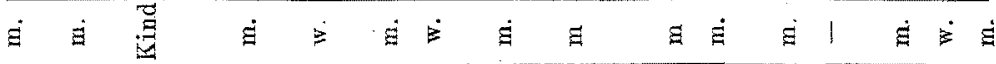
$\because$ 电1

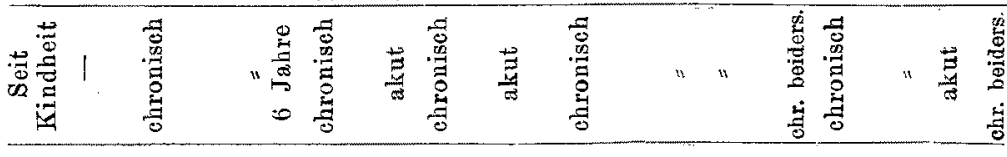

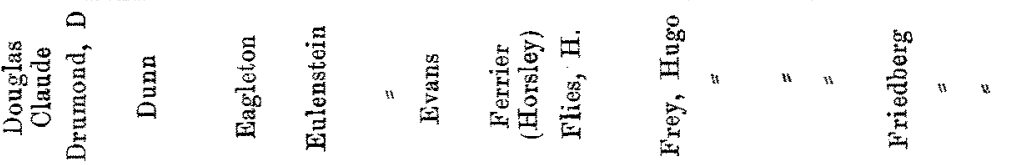

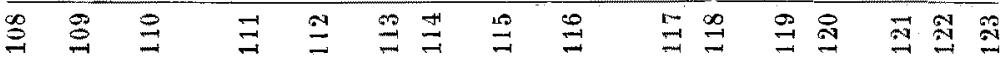




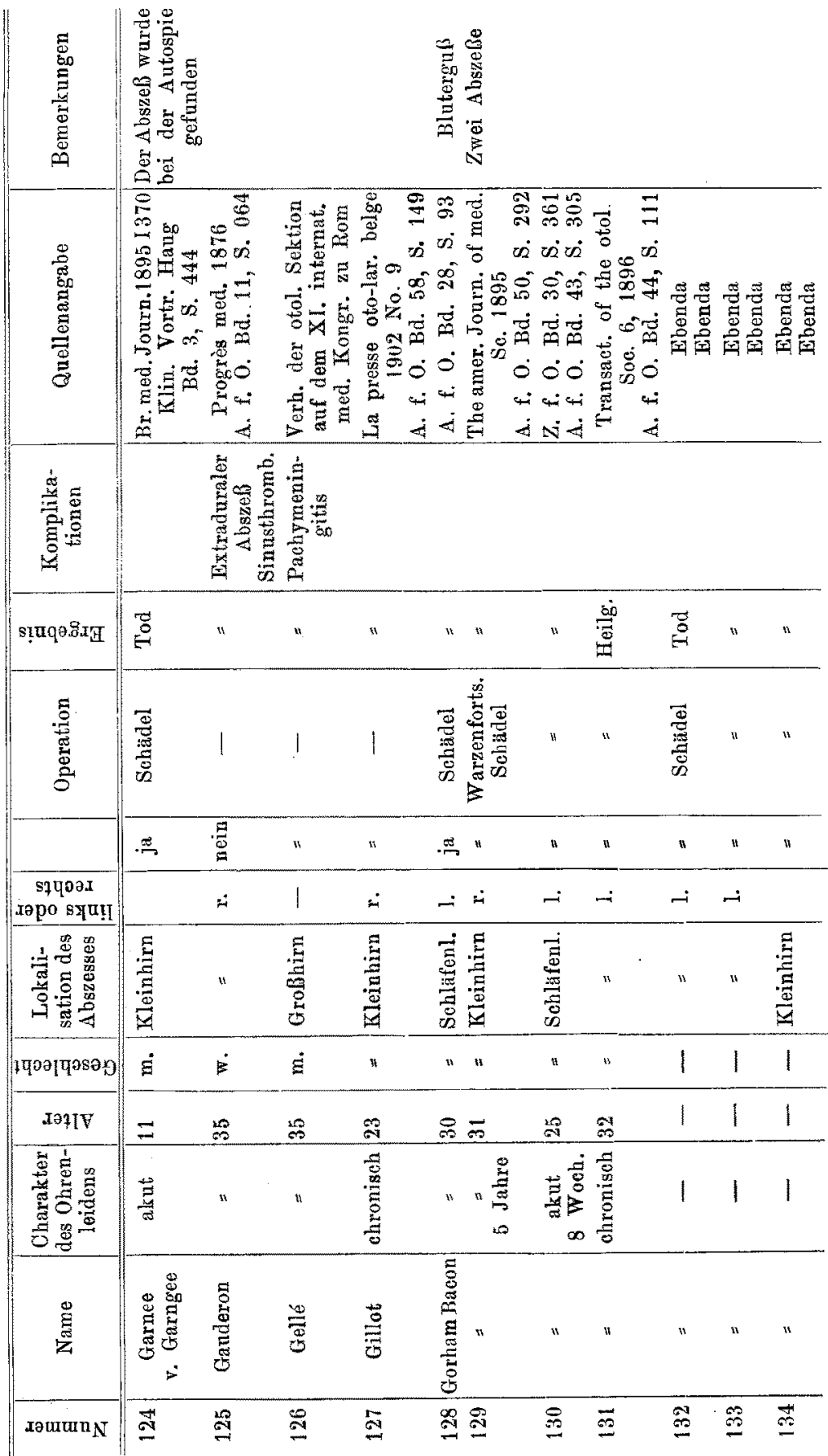



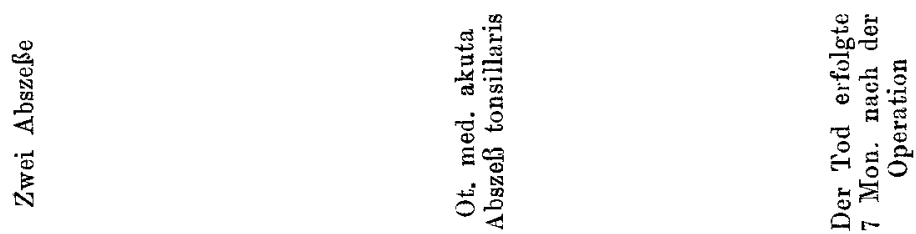

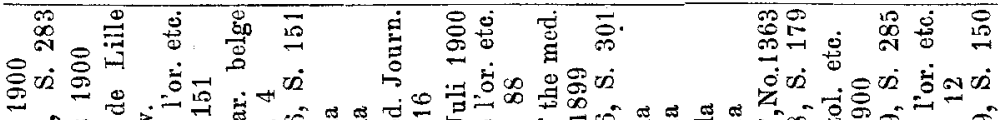

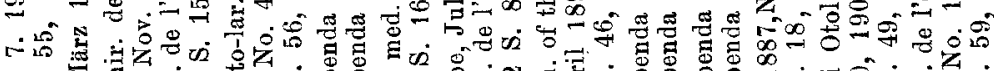

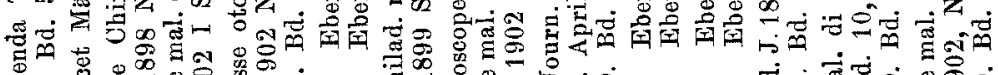

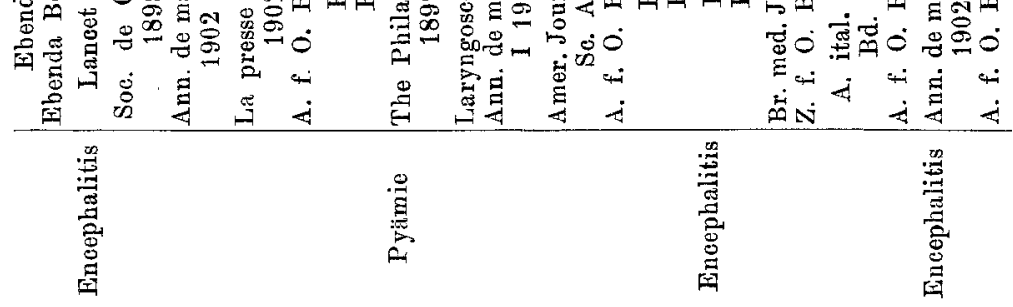

\begin{tabular}{|c|c|c|c|c|c|c|c|c|c|c|}
\hline 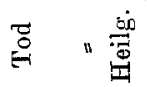 & " & " & : & $\stackrel{\dot{s}}{\overrightarrow{0}}$ & 莡 & $"$ & " & 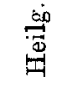 & $\stackrel{\square}{*}$ & " \\
\hline
\end{tabular}

\begin{tabular}{|c|c|c|c|c|c|c|c|c|c|}
\hline 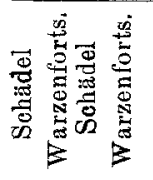 & 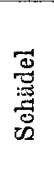 & 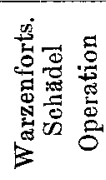 & 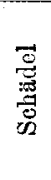 & 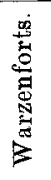 & " & 1 & 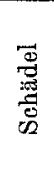 & 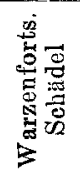 & " \\
\hline. & $"$ & $"$ & $"$ & $"$ & $"$ & $\infty$ & $\stackrel{\Phi}{.}$ & " & $"$ \\
\hline$\dot{4} \quad \dot{1} \mid$ & ! & $\dot{-}$ & نت & 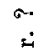 & $\dot{i}$ & -7 & $\therefore$ & $\therefore$ & - \\
\hline
\end{tabular}

\begin{tabular}{|c|c|c|c|c|c|c|c|c|c|c|c|}
\hline & 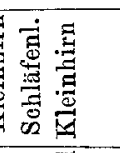 & 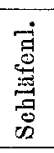 & " & 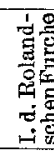 & 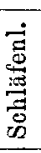 & 晃 & " & " & 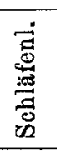 & 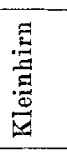 & 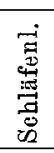 \\
\hline$\geqslant$ & $\approx \widetilde{\Xi}$ & $\dot{\Xi}$ & " & $"$ & 1 & $\exists$ & $"$ & 1 & $\dot{\varepsilon}$ & $\dot{E}$ & $\dot{\Xi}$ \\
\hline ศิ & $=+$ & 早 & ฐึ & 1 & 1 & $\stackrel{\sim}{\circ}$ & $\stackrel{\mathcal{D}}{\mathrm{N}}$ & 1 & \% & $\stackrel{\infty}{0}$ & 1 \\
\hline 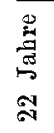 & 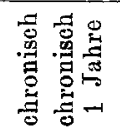 & 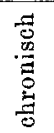 & " & $\frac{\vec{E}}{\frac{\vec{E}}{\omega}}$ & " & 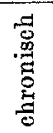 & " & $"$ & $\stackrel{\vec{\Xi}}{\Xi}$ & 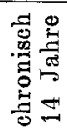 & 1 \\
\hline " & 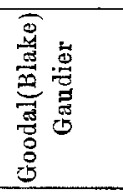 & 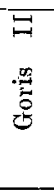 & $\begin{array}{l}\Xi \\
\Xi\end{array}$ & 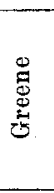 & 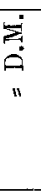 & 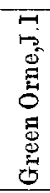 & 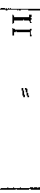 & $\Xi$ & 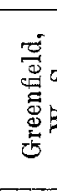 & 蛋 & $\stackrel{\overrightarrow{0}}{\vec{B}}$ \\
\hline & $\underset{\sim}{\infty}$ & $\begin{array}{l}\infty \\
\wp \\
\Leftrightarrow\end{array}$ & $\stackrel{90}{9}$ & 㝵 & $\exists$ & $\underset{\forall}{\Psi}$ & $\exists$ & $\Xi$ & $\frac{18}{15}$ & 吕 & E \\
\hline
\end{tabular}

